\title{
MARTIN BOUNDARY OF A KILLED RANDOM WALK ON A HALF-SPACE
}

\author{
IRINA IGNATIOUK-ROBERT
}

\begin{abstract}
A complete representation of the Martin boundary of killed random walks on a half-space $\mathbb{Z}^{d-1} \times \mathbb{N}^{*}$ is obtained. In particular, it is proved that the corresponding Martin boundary is homemorphic to the half-sphere $\mathcal{S}_{+}^{d}=\left\{z \in \mathbb{R}^{d-1} \times \mathbb{R}_{+}:|z|=1\right\}$. The method is based on a combination of ratio limits theorems and large deviation techniques.
\end{abstract}

\section{INTRODUCTION}

The concept of Martin compactification was first introduced for Brownian motion by Martin [16]. For countable Markov chains with discrete time, the abstract construction of the Martin compactification was given by Doob [9] and Hunt [12, see also Dynkin [10] and Rogers and Williams [20. The main results of this theory are the following :

For a transient Markov chain $(Z(n))$ on a countable set $E$ having Green's function $G\left(z, z^{\prime}\right)$, the Martin kernel $K\left(z, z^{\prime}\right)$ is defined by

$$
K\left(z, z^{\prime}\right)=G\left(z, z^{\prime}\right) / G\left(z_{0}, z^{\prime}\right)
$$

where $z_{0}$ is a given reference point in $E$. A sequence $z_{n} \in E$ is said to converge to a point on a Martin boundary $\partial E_{M}$ of $E$ if it leaves every finite subset on $E$ and the sequence of functions $K\left(\cdot, z_{n}\right)$ converges point-wise. According to this definition, the Martin compactification $E_{M}$ is the unique smallest compactification of the set $E$ for which the Martin kernels $K(z, \cdot)$ extend continuously.

The minimal Martin boundary $\partial_{m} E_{M}$ is the set of all those $\gamma \in \partial E_{M}$ for which the function $K(\cdot, \gamma)$ is minimal harmonic. Recall that a function $h: E \rightarrow \mathbb{R}_{+}$is harmonic for $(Z(n))$ if $\mathbb{E}_{z}(h(Z(1)))=h(z)$ for all $z \in E$. A harmonic function $h: E \rightarrow \mathbb{R}_{+}$is minimal if the inequality $0 \leq h^{\prime} \leq h$ for any other harmonic function $h^{\prime}$ implies that $h^{\prime}=c h$ with some $c>0$.

By the Poisson-Martin representation theorem, for every non-negative harmonic function $h$ there exists a positive Borel measure $\nu$ on $\partial_{m} E_{M}$ such that

$$
h(z)=\int_{\partial_{m} E_{M}} K(z, \eta) d \nu(\eta)
$$

By Convergence theorem, the sequence $(Z(n))$ converges $\mathbb{P}_{z}$ almost surely for every $z \in E$ to a $\partial_{m} E_{M}$ valued random variable. The Martin boundary provides therefore the non-negative harmonic functions and shows how the Markov chain $(Z(n))$ goes to infinity. A good introduction to the theory of Martin compactification for

Date: August 3, 2018.

1991 Mathematics Subject Classification. Primary 60F10; Secondary 60J15, 60K35.

Key words and phrases. Martin boundary.Sample path large deviations. Random walk. 
countable Markov chains and a wide literature of related results is given in the book of Woess 24].

An explicit description of the Martin compactification is usually a non-trivial problem. A large number of results in this domain has been obtained for homogeneous random walks. For homogeneous random walks on $\mathbb{Z}^{d}$, the Martin boundary was identified by Ney and Spitzer [18. They considered an irreducible random walk $(Z(t))$ on $\mathbb{Z}^{d}$ with transition probabilities $p\left(z, z^{\prime}\right)=\mu\left(z^{\prime}-z\right)$ and a non zero mean, for which the jump generating function

$$
\varphi(a) \doteq \sum_{z \in \mathbb{Z}^{d}} \mu(z) \exp (a \cdot z)
$$

is finite in a neighborhood of the set

$$
D \doteq\left\{a \in \mathbb{R}^{d}: \varphi(a) \leq 1\right\} .
$$

For such a random walk, the set $D$ is compact and convex, the gradient $\nabla \varphi(a)$ exists everywhere on $\mathbb{R}^{d}$ and does not vanish on the boundary $\partial D \doteq\{a: \varphi(a)=1\}$, and the mapping

$$
q(a)=\nabla \varphi(a) /|\nabla \varphi(a)|
$$

determines a homeomorphism between $\partial D$ and the unit sphere $\mathcal{S}^{d}$ in $\mathbb{R}^{d}$ (see [1]). Using exponential change of measure and the local limit theorem, Ney and Spitzer calculated the exact asymptotics of the Green's function

$$
G\left(z, z^{\prime}\right)=\sum_{n} \mathbb{P}_{z}\left(Z(n)=z^{\prime}\right)
$$

and deduced that for any $a \in \partial D$, and any sequence of points $z_{n} \in \mathbb{Z}^{d}$ with $\lim _{n}\left|z_{n}\right| \rightarrow \infty$ and $\lim _{n} z_{n} /\left|z_{n}\right|=q(a)$,

$$
G\left(z, z_{n}\right) / G\left(0, z_{n}\right) \rightarrow \exp (a \cdot z) \text { as } n \rightarrow \infty, \quad \forall z \in \mathbb{Z}^{d} .
$$

Hence, for the homogeneous random walk on $\mathbb{Z}^{d}$, a sequence of points $z_{n} \in \mathbb{Z}^{d}$ with $\lim _{n}\left|z_{n}\right|=\infty$ converges to a point on the Martin boundary if and only if the sequence $z_{n} /\left|z_{n}\right|$ converges to a point on a unit sphere in $\mathbb{R}^{d}$. The Martin compactification of the lattice $\mathbb{Z}^{d}$ determined by the homogeneous random walk is therefore homeomorphic to the closure of the set $\left\{w=z /(1+|z|): z \in \mathbb{Z}^{d}\right\}$ in $\mathbb{R}^{d}$.

For a wide literature of results where the Martin boundary was identifies for more general homogeneous Markov chains such as random walks on free groups, hyperbolic graphs, Cartesian products we refer to the book of Woess 24 and the references therein.

Only few results identify the Martin boundary for non-homogeneous Markov chains. For random walks on non-homogeneous trees the Martin boundary was described by Cartier [4. Doney [ 8 identified the harmonic functions and the Martin boundary of a homogeneous random walk $(Z(n))$ on $\mathbb{Z}$ killed on the negative halfline $\{z: z<0\}$. For space-time random walk $S(n)=(Z(n), n)$ for a homogeneous random walk $Z(n)$ on $\mathbb{Z}$ killed on the negative half-line $\{z: z<0\}$ the Martin boundary was described by Alili and Doney [1. These results use special onedimensional structure of the process. For Brownian motion on a half-space, the Martin boundary was obtained in the book of Doob 9 ] by using an explicit form of the Green's function. Kurkova and Malyshev [15] described the Martin boundary 
for random walks on $\mathbb{Z} \times \mathbb{N}$ and on $\mathbb{Z}_{+}^{2}$ for which the only non-zero transitions in the interior of the domain are on the nearest neighbors: $p\left(z, z \pm e_{i}\right)=\mu\left( \pm e_{i}\right)$ with $e_{1}=(1,0)$ and $e_{2}=(0,1)$. For such random walks, the jump generating function is defined by $\varphi(x, y)=\mu\left(e_{1}\right) x+\mu\left(-e_{1}\right) x^{-1}+\mu\left(e_{2}\right) y+\mu\left(-e_{2}\right) y^{-1}$ and the equation $x y(1-\varphi(x, y))=0$ determines an elliptic curve $\mathbf{S}$ which is homeomorphic to the torus. To identify the Martin boundary, a functional equation is derived for the generating function of the Green's function and the asymptotics of the Green's function are calculated by using the methods of complex analysis on the elliptic curve $\mathbf{S}$. Such a method seems to be unlikely to apply in more general situations, for higher dimensions or when the jump sizes are arbitrary, because the proof is based on the geometrical properties of the elliptic curve $\mathbf{S}$.

In the present paper, we identify the Martin boundary for a random walk $Z_{+}(t)$ on $\mathbb{Z}^{d}$ which is killed when leaving the half-space $\mathbb{Z}^{d-1} \times \mathbb{N}^{*}$ where $\mathbb{N}^{*}=\mathbb{N} \backslash\{0\}$. This is a substochastic Markov process on $\mathbb{Z}^{d-1} \times \mathbb{N}^{*}$ with transition matrix

$$
\left(p\left(z, z^{\prime}\right)=\mu\left(z^{\prime}-z\right) ; z, z^{\prime} \in \mathbb{Z}^{d-1} \times \mathbb{N}^{*}\right)
$$

where $\mu$ is a probability measure on $\mathbb{Z}^{d}$. Such a Markov process dies when the homogeneous random walk $Z(t)$ exits from $\mathbb{Z}^{d-1} \times \mathbb{N}^{*}$ and is identical to $Z(t)$ until the first time when $Z(t) \notin \mathbb{Z}^{d-1} \times \mathbb{N}^{*}$. The Green function

$$
G_{+}\left(z, z^{\prime}\right)=\sum_{n} \mathbb{P}_{z}\left(Z_{+}(n)=z^{\prime}\right)
$$

is the mean number of visits to the point $z^{\prime}$ starting from $z$ before hitting the set $\mathbb{Z}^{d-1} \times(-\mathbb{N})$. The homogeneous random walk $Z(t)$ is assumed to satisfy the following conditions

(A) The Markov chain $Z(t)$ is irreducible and has a non zero mean

$$
m \doteq \sum_{z \in \mathbb{Z}^{d}} z \mu(z) \neq 0,
$$

the last coordinate $Y(t)$ of $Z(t)$ is an aperiodic random walk on $\mathbb{Z}$ and the jump generating function $\varphi$ defined by (1.1) is finite everywhere on $\mathbb{R}^{d}$.

For such a killed random walk, the limit of the sequence of functions

$$
G_{+}\left(\cdot, z_{n}\right) / G_{+}\left(z_{0}, z_{n}\right)
$$

can be identified by using the results of Borovkov 3 for those sequences $z_{n} \in$ $Z^{d-1} \times \mathbb{N}$ for which $\lim _{n \rightarrow \infty} z_{n} /\left|z_{n}\right|=(u, v) \in \mathbb{R}^{d-1} \times \mathbb{R}_{+}$with $v \neq 0$. The most difficult case is when $\lim _{n \rightarrow \infty} z_{n} /\left|z_{n}\right| \in \mathbb{R}^{d-1} \times\{0\}$, in particular if

$$
z_{n}=\left(x_{n}, y\right) \in \mathbb{Z}^{d-1} \times\{y\}
$$

for all $n \in \mathbb{N}$. Here, the results of Borovkov 3 do not work. The analytical method of Kurkova and Malyshev do not seem to apply at all because one should consider the curve determined by the equation

$$
\sum_{k=\left(k_{1}, \ldots, k_{d}\right) \in \mathbb{Z}^{d}} \mu(k) z_{1}^{k_{1}} \cdot \ldots \cdot z_{d}^{k_{d}}=1
$$

with an infinite number of terms at the left-hand side.

While the problem of Martin boundary identification of these killed random walks is interesting in its own right, our methods also apply to the setting of Ney and Spitzer [18] and lead to another simple proof of their theorem (see Section 7 below). 
The technical results of the present paper are important for the identification of the Martin boundary of random walks on the half-space with reflected boundary conditions on the hyper-plane $\mathbb{Z}^{d-1} \times\{0\}$, see Ignatiouk [14].

To formulate our result, it is convenient to introduce the following notations : $q \rightarrow a(q)$ is the inverse mapping of the function (1.2) and, for $q \in \mathbb{R}^{d} \backslash\{0\}$, $a(q) \doteq a(q /|q|)$ is the unique point on the boundary $\partial D$ of the set $D$ where the normal cone to $D$ contains the vector $q$. According to this definition, for $q \neq 0$,

$$
\sup _{a \in D} a \cdot q=a(q) \cdot q>a \cdot q, \quad \forall a \in D \backslash\{a(q)\}
$$

(see Rockafellar [19]). Define also the half-sphere $\mathcal{S}_{+}^{d} \doteq\left\{q \in \mathbb{R}^{d-1} \times \mathbb{R}_{+}:|q|=1\right\}$ with $\mathbb{R}_{+}=\left[0,+\infty\left[\right.\right.$ and the sets $\partial_{+} D=\left\{a \in \partial D: q(a) \in \mathbb{R}^{d-1} \times \mathbb{R}_{+}\right\}$and

$$
\partial_{0} D=\left\{a \in \partial D: q(a) \in \mathbb{R}^{d-1} \times\{0\}\right\} \subset \partial_{+} D .
$$

Denote by $X(t)$ [resp. $Y(t)]$ the vector of $d-1$ first coordinates [resp. the last coordinate ] of the vector $Z(t) \in \mathbb{R}^{d}$ and let $\tau$ be the killing time of the process $\left(Z_{+}(t)\right)$ :

$$
\tau=\inf \{t: Y(t) \leq 0\} .
$$

The variables $X_{+}(t)$ and $Y_{+}(t)$ are defined in a similar way for the vector $Z_{+}(t)$. According to the definition of the process $Z_{+}(t),\left(X_{+}(t), Y_{+}(t)\right)=(X(t), Y(t))$ for $t<\tau$ and $Z(\tau)=(X(\tau), Y(\tau)) \in \mathbb{Z}^{d-1} \times(-\mathbb{N})$ is the point where the process $Z_{+}(t)$ dies. For every $a \in \partial_{+} D$, the function $h_{a,+}$ on $\mathbb{Z}^{d-1} \times \mathbb{N}^{*}$ is defined by

$$
h_{a,+}(z) \doteq \begin{cases}y \exp (a \cdot z)-\mathbb{E}_{z}(Y(\tau) \exp (a \cdot Z(\tau)), \tau<+\infty) & \text { if } a \in \partial_{0} D, \\ \exp (a \cdot z)-\mathbb{E}_{z}(\exp (a \cdot Z(\tau)), \tau<+\infty) & \text { if } a \in \partial_{+} D \backslash \partial_{0} D .\end{cases}
$$

The main result of the paper is the following theorem.

Theorem 1. Under the hypotheses (A), the following assertions hold:

1) The constant multiples of the functions $h_{a,+}$ with $a \in \partial_{+} D$ are the only minimal harmonic functions of the Markov process $\left(Z_{+}(t)\right)$.

2) For any $q \in \mathcal{S}_{+}^{d}$ and any sequence of points $z_{n} \in \mathbb{Z}^{d-1} \times \mathbb{N}^{*}$ with $\lim _{n}\left|z_{n}\right|=\infty$ and $\lim _{n} z_{n} /\left|z_{n}\right|=q$,

$$
\lim _{n \rightarrow \infty} G_{+}\left(z, z_{n}\right) / G_{+}\left(z_{0}, z_{n}\right)=h_{a(q),+}(z) / h_{a(q),+}\left(z_{0}\right)
$$

for all $z=(x, y) \in \mathbb{Z}^{d-1} \times \mathbb{N}^{*}$.

From this theorem it follows the following statement.

Corollary 1.1. Under the hypotheses (A), the following assertions hold:

1) A sequence of points $z_{n} \in \mathbb{Z}^{d-1} \times \mathbb{N}^{*}$ with $\lim _{n}\left|z_{n}\right|=+\infty$ converge to a point of the Martin boundary for the Markov process $Z_{+}(t)$ if and only if $z_{n} /\left|z_{n}\right| \rightarrow q$ for some point $q$ on the half-sphere $\mathcal{S}_{+}^{d}$.

2) The full Martin compactification of the half-space $\mathbb{Z}^{d-1} \times \mathbb{N}^{*}$ is homeomorphic to the closure of the set $\left\{w=z /(1+|z|): z \in \mathbb{Z}^{d-1} \times \mathbb{N}^{*}\right\}$ in $\mathbb{R}^{d}$.

3) The minimal Martin boundary coincides with the whole Martin boundary.

The proof of Theorem 11 uses the properties of Markov-additive processes. A Markov process $(A(t), M(t))$ on a countable set $\mathbb{Z}^{d} \times E$ is said to be Markov-additive if its transition probabilities are invariant with respect to the shifts on $x \in \mathbb{Z}^{d}$ :

$$
\mathbb{P}_{(x, y)}\left((A(t), M(t))=\left(x^{\prime}, y^{\prime}\right)\right)=\mathbb{P}_{(0, y)}\left((A(t), M(t))=\left(x^{\prime}-x, y^{\prime}\right)\right),
$$


for all $(x, y),\left(x^{\prime}, y^{\prime}\right) \in \mathbb{Z}^{d} \times E$. For such a Markov process, $A(t)$ is called an additive part and $M(t)$ is its Markovian part. With this definition, the Markov process $\left(Z_{+}(t)=\left(X_{+}(t), Y_{+}(t)\right)\right)$ is Markov-additive with an additive part $X_{+}(t)$ on $\mathbb{Z}^{d-1}$ and a Markovian part $Y_{+}(t)$ on $E=\mathbb{N}^{*}$.

The first assertion of Theorem 1 is proved by using the arguments of ChoquetDeny theory adapted for Markov-additive processes. The main steps of the proof of the second assertion are the following.

Under some general assumptions on the Markov-additive process $(A(t), M(t))$ on $\mathbb{Z}^{d} \times E$, it is proved that the Green's function

$$
\mathcal{G}\left((k, y),\left(l, y^{\prime}\right)\right)=\sum_{n} \mathbb{P}_{(k, y)}\left((A(n), M(n))=\left(l, y^{\prime}\right)\right)
$$

satisfies the following property : if for a sequence $z_{n}=\left(x_{n}, y_{n}\right) \in \mathbb{Z}^{d} \times E$ converging to infinity, the inequality

$$
\lim _{n \rightarrow \infty} \frac{1}{\left|z_{n}\right|} \log \mathcal{G}\left((0, y), z_{n}\right) \geq 0
$$

holds for some $y \in E$ then also

$$
\lim _{n \rightarrow \infty} \mathcal{G}\left(\left(x+x^{\prime}, y\right), z_{n}\right) / \mathcal{G}\left(\left(x+x^{\prime \prime}, y\right), z_{n}\right)=1, \quad \forall x, x^{\prime} \in \mathbb{Z}^{d}, y \in E
$$

for all $(x, y) \in \mathbb{Z}^{d} \times E$ and all those $x^{\prime}, x^{\prime \prime} \in \mathbb{Z}$ for which

$$
\inf _{y \in E} \min \left\{\mathbb{P}_{(0, y)}\left(A(k)=x^{\prime}, M(k)=y\right), \mathbb{P}_{(0, y)}\left(A(k)=x^{\prime \prime}, M(k)=y\right)\right\}>0 .
$$

This is an extension of an intermediate technical result of Foley and McDonald [7]) obtained for a different purpose in a more restricted context for Markov-additive processes with a one-dimensional additive part and for a sequence of the form $z_{n}=(n, y)$.

For the random walk $\left(Z_{+}(t)\right)$, this result implies that

$$
\lim _{n \rightarrow \infty} G_{+}\left(\left(x+\hat{k} x^{\prime}, y\right), z_{n}\right) / G_{+}\left((x, y), z_{n}\right)=1, \quad \forall x, x^{\prime} \in \mathbb{Z}^{d-1}, y \in \mathbb{N}
$$

whenever

$$
\lim _{n \rightarrow \infty} \frac{1}{\left|z_{n}\right|} \log G_{+}\left(z, z_{n}\right)=0 \quad \text { for some } \quad z \in \mathbb{Z}^{d-1} \times \mathbb{N}^{*}
$$

where $\hat{k}$ denotes the period of the random walk $(Z(t))$.

The second important tool of our proof is the use of large deviation techniques. With the aid of Mogulskii's theorem (see Dembo and Zeitouni [6]), we show that the family of scaled processes $Z_{+}^{\varepsilon}(t)=\varepsilon Z_{+}([t / \varepsilon])$ satisfies sample path large deviation principle. The inequality (1.5) is obtained for any $z \in \mathbb{Z}^{d-1} \times \mathbb{N}^{*}$ and any sequence of points $z_{n} \in \mathbb{Z}^{d-1} \times \mathbb{N}^{*}$ when

$$
\lim _{n \rightarrow \infty} z_{n} /\left|z_{n}\right|=q(0) \in \partial_{+} D
$$

by using the lower large deviation bound.

Finally, we use the results of Doney [8] and the integral representation of the harmonic functions to show that for every $a=(\alpha, \beta) \in \partial_{+} D$ with $\alpha \in \mathbb{R}^{d-1}$ and $\beta \in \mathbb{R}$, the constant multiples of the function $h_{a,+}$ are the only non-negative harmonic functions satisfying the equality

$$
h\left(x+\hat{k} x^{\prime}, y\right)=\exp \left(\hat{k} \alpha \cdot x^{\prime}\right) h(x, y), \quad \forall x, x^{\prime} \in \mathbb{Z}^{d-1}, y \in \mathbb{N} .
$$


The last result and the equality (1.4) are used to get the convergence (1.3) when $q=q(0) \in \mathbb{R}^{d-1} \times \mathbb{R}_{+}$. An exponential change of measure with a parameter $a(q)$ extends this result for an arbitrary $q \in \mathcal{S}_{+}^{d}$.

Before proving our results, an example where the functions $h_{a,+}$ have an explicit form is given.

\section{An EXPLicit REPRESENTATION OF THE FUnCtions $h_{a,+}$}

In this section, we calculate explicitly the functions $h_{a,+}$ for $a \in \partial_{+} D$ in a particular case when $\mu(z)=0$ for all $z=(x, y) \in \mathbb{Z}^{d-1} \times \mathbb{Z}$ with $y<-1$. In this classical situation, the last vertical component $(Y(t))$ of the random walk $(Z(t))$ is left continuous, i.e. the value of a downward jump is -1 .

Let $\pi: \mathbb{R}^{d} \rightarrow \mathbb{R}^{d}$ be the orthogonal projection to the hyper-plane $\mathbb{R}^{d-1} \times\{0\}$. For a point $a \in \partial_{+} D$ with $q(a) \in S_{+}^{d}$ we denote by $\bar{a}$ the unique point on the boundary $\partial D$ with $\left.\left.q(\bar{a}) \in S^{d} \cap\left(\mathbb{R}^{d} \times\right]-\infty, 0\right]\right)$ for which $\pi(a)=\pi(\bar{a})$. Clearly, $a=\bar{a}$ if and only if $a \in \partial_{0} D$.

Proposition 2.1. Suppose that $\mu(z)=0$ for all $z=(x, y) \in \mathbb{Z}^{d}$ with $y<-1$, then under the hypotheses (A), for every $a \in \partial_{+} D$,

$$
h_{a,+}(z)=\left\{\begin{array}{ll}
\exp (a \cdot z)-\exp (\bar{a} \cdot z) & \text { if } a \notin \partial_{0} D, \\
y \exp (a \cdot z) & \text { if } a \in \partial_{0} D,
\end{array} \quad \forall z=(x, y) \in \mathbb{Z}^{d} \times \mathbb{N}^{*} .\right.
$$

Proof. Indeed, if $\mu(z)=0$ for all $z=(x, y) \in \mathbb{Z}^{d-1} \times \mathbb{Z}$ with $y<-1$ then almost surely $Y(\tau)=0$ and consequently, for every $a=(\alpha, \beta) \in \partial_{+} D$ with $\alpha \in \mathbb{R}^{d-1}$ and $\beta \in \mathbb{R}$,

$$
h_{a,+}(z)= \begin{cases}\exp (a \cdot z)-\mathbb{E}_{z}(\exp (\alpha \cdot X(\tau)) ; \tau<+\infty) & \text { if } a \in \partial_{+} D \backslash \partial_{0} D \\ y \exp (a \cdot z) & \text { if } a \in \partial_{0} D\end{cases}
$$

Furthermore, for $a \in \partial_{+} D \backslash \partial_{0} D$, the quantity

$$
\exp (-\bar{a} \cdot z) \mathbb{E}_{z}(\exp (\alpha \cdot X(\tau)) ; \tau<+\infty)=\mathbb{E}_{z}(\exp (\bar{a} \cdot(Z(\tau)-z)), \tau<+\infty)
$$

is equal to the probability that the twisted homogeneous random walk $\bar{Z}(t)$ on $\mathbb{Z}^{d}$ with transition probabilities

$$
p_{\bar{a}}\left(z, z^{\prime}\right)=\exp \left(\bar{a} \cdot\left(z^{\prime}-z\right)\right) \mu\left(z^{\prime}-z\right),
$$

starting from $z$ ever hits the boundary hyper-plane $\mathbb{Z}^{d-1} \times\{0\}$. Since the last coordinate of the mean

$$
\mathbb{E}_{0}(\bar{Z}(1))=\sum_{z \in \mathbb{Z}^{d-1} \times \mathbb{Z}} z \mu(z) \exp (\bar{a} \cdot z)=\nabla \varphi(\bar{a})
$$

is negative then for every starting point $z \in \mathbb{Z}^{d-1} \times \mathbb{N}^{*}$, this hitting probability is equal to 1 and consequently, (2.1) holds.

\section{General properties of Markov-additive processes}

In this section we describe minimal harmonic function of Markov-additive processes and prove the ratio limit theorem. Before to prove these results we recall the definition of Markov-additive processes, the corresponding Feynman-Kac transform and its spectral radius. 
A Markov process $(A(t), M(t))$ on a countable set $\mathbb{Z}^{d} \times E$ with transition probabilities $p\left((x, y),\left(x^{\prime}, y^{\prime}\right)\right)$ is called Markov-additive if

$$
p\left((x, y),\left(x^{\prime}, y^{\prime}\right)\right)=p\left((0, y),\left(x^{\prime}-x, y^{\prime}\right)\right)
$$

for all $x, x^{\prime} \in \mathbb{Z}^{d}, y, y^{\prime} \in E$. The first component $A(t)$ is an additive part of the process $(A(t), M(t))$, and $M(t)$ is its Markovian part. The Markovian part $M(t)$ is a Markov chain on $E$ with transition probabilities

$$
P\left(y, y^{\prime}\right)=\sum_{x \in \mathbb{Z}^{d}} p\left((0, y),\left(x, y^{\prime}\right)\right) .
$$

For $\alpha \in \mathbb{R}^{d}$, an infinite matrix $\mathcal{P}(\alpha)=\left(\mathcal{P}\left(\alpha, y, y^{\prime}\right), y, y^{\prime} \in E\right)$ with

$$
\mathcal{P}\left(\alpha, y, y^{\prime}\right)=\mathbb{E}_{(0, y)}\left(\exp (\alpha \cdot A(1)) ; M(1)=y^{\prime}\right)
$$

is called Feynman-Kac transform of the Markov-additive process $(A(t), M(t))$. The $n$-th iterate $\mathcal{P}^{n}(\alpha)=\left(\mathcal{P}^{(n)}\left(\alpha, y, y^{\prime}\right), y, y^{\prime} \in E\right)$ of the matrix $\mathcal{P}(\alpha)$ is given by

$$
\mathcal{P}^{(n)}\left(\alpha, y, y^{\prime}\right)=\mathbb{E}_{(0, y)}\left(\exp (\alpha \cdot A(n)) ; M(n)=y^{\prime}\right), \quad y, y^{\prime} \in E .
$$

If the Markovian part $(M(t))$ is irreducible then the matrix $\mathcal{P}(\alpha)$ is also irreducible. In this case, for every $\lambda \in \mathbb{R}$, the series

$$
G_{\lambda}\left(\alpha, y, y^{\prime}\right) \doteq \sum_{n=0}^{\infty} e^{-\lambda n} \mathcal{P}^{(n)}\left(\alpha, y, y^{\prime}\right)
$$

converge or diverge simultaneously for all $y, y^{\prime} \in E$, and the limit

$$
\lambda(\alpha)=\limsup _{n} \frac{1}{n} \log \mathcal{P}^{(n)}\left(\alpha, y, y^{\prime}\right)
$$

does not depend on $y, y^{\prime} \in E$ (see [22]). The quantity $e^{\lambda(\alpha)}$ is usually called spectral radius and $e^{-\lambda(\alpha)}$ is the convergence parameter of the transform matrix $\mathcal{P}(\alpha)$, it is a common radius of convergence of the series (3.1). According to this definition, $\lambda(\alpha)=+\infty$ if $\mathcal{P}\left(\alpha, y, y^{\prime}\right)=+\infty$ for some $y, y^{\prime} \in E$.

3.1. Minimal harmonic functions. Recall that a non-negative function $h$ on $\mathbb{Z}^{d} \times E$ is harmonic for the Markov process $(A(t), M(t))$ if $\mathbb{E}_{z}(h(A(t), M(t)))=h(z)$ for all $z \in \mathbb{Z}^{d} \times E$. A non-zero harmonic function $h \geq 0$ is minimal if for any nonzero harmonic function $h^{\prime} \geq 0$, the inequality $h^{\prime} \leq h$ implies that $h^{\prime}=c h$ with some constant $c>0$.

Proposition 3.1. Suppose that the Markovian part $(M(t))$ is irreducible and that for every $x \in \mathbb{Z}^{d}$, there are $n \in \mathbb{N}$ and $\theta>0$ such that $p^{(n)}((0, y),(x, y)) \geq \theta$ for all $y \in E$. Then every non-zero minimal harmonic function $h$ of the Markov process $(A(t), M(t))$ is of the form

$$
h(x, y)=e^{\alpha \cdot x} h(0, y)>0, \quad \forall(x, y) \in \mathbb{Z}^{d} \times E
$$

with some $\alpha \in \mathbb{R}^{d}$ satisfying the inequality $\lambda(\alpha) \leq 0$.

Proof. The proof of this proposition uses the arguments similar to that of Choquet and Deny theorem (see Woess [24]). 
Let $h(x, y) \geq 0$ be a harmonic function. Then for a unit vector $e_{i} \in \mathbb{Z}^{d}$, the function $h_{i}(x, y)=h\left(x+e_{i}, y\right)$ is also harmonic and under the hypotheses of our proposition, there exist $n_{i} \in \mathbb{N}$ and $\theta>0$ such that

$$
\begin{aligned}
h(x, y) & =\sum_{\left(x^{\prime}, y^{\prime}\right)} p^{\left(n_{i}\right)}\left((x, y),\left(x^{\prime}, y^{\prime}\right)\right) h\left(x^{\prime}, y^{\prime}\right) \geq p^{\left(n_{i}\right)}\left((x, y),\left(x+e_{i}, y\right)\right) h\left(x+e_{i}, y\right) \\
& \geq \theta h_{i}(x, y)
\end{aligned}
$$

for all $(x, y) \in \mathbb{Z}^{d} \times E$. If the harmonic function $h$ is minimal, the last inequality implies that $h_{i}=c_{i} h$ for some $c_{i}>0$. Using the equality $h=c_{i} h_{i}$ for every unit vector $e_{i} \in \mathbb{Z}^{d}, i=1, \ldots, d$, and letting $\alpha_{i}=\ln c_{i}$ we obtain (3.2). Moreover, since the function $h$ is harmonic and since (3.2) holds,

$$
h(0, y)=\sum_{y^{\prime} \in E} \mathcal{P}^{(n)}\left(\alpha, y, y^{\prime}\right) h\left(0, y^{\prime}\right) \geq \mathcal{P}^{(n)}\left(\alpha, y, y^{\prime}\right) h\left(0, y^{\prime}\right)
$$

for all $y, y^{\prime} \in E$ and for all $n \in \mathbb{N}$. Under the hypotheses of our proposition, the matrix $\mathcal{P}(\alpha)$ is irreducible and hence, the last inequality implies that $h(0, y)>0$ for all $y \in E$ and

$$
\lambda(\alpha)=\limsup _{n} \frac{1}{n} \log \mathcal{P}^{(n)}\left(\alpha, y, y^{\prime}\right) \leq 0
$$

whenever $h \not \equiv 0$. Proposition 3.1 is therefore proved.

3.2. Ratio limit theorem. Throughout this section, $\mathcal{Z}(t)=(A(t), M(t))$ denotes a Markov-additive process on $\mathbb{Z}^{d} \times E$ with an additive part $(A(t))$ on $\mathbb{Z}^{d}$ and a Markovian part $(M(t))$ on $E \subset \mathbb{Z}^{k}$. Green's function of the Markov process $\mathcal{Z}(t)$ is denoted by $\mathcal{G}\left(z, z^{\prime}\right)$. The following conditions are assumed to be satisfied.

(A1) The Markov-additive process $\mathcal{Z}(t)=(A(t), M(t))$ is irreducible.

(A2) The function

$$
\hat{\varphi}(a)=\sup _{z \in \mathbb{Z}^{d} \times E} \mathbb{E}_{z}(\exp (a \cdot(\mathcal{Z}(1)-z)))
$$

is finite in a neighborhood of zero.

Remark that the Markov-additive process $\mathcal{Z}(t)=(A(t), M(t))$ is not necessarily stochastic : in some points $z=(x, y) \in \mathbb{Z}^{d} \times E$, the transition matrix can be strictly substochastic.

Proposition 3.2. Suppose that the Markov-additive process $\mathcal{Z}(t)=(A(t), M(t))$ is transient and satisfies the hypotheses (A1), (A2) and let a sequence of points $z_{n} \in \mathbb{Z}^{d} \times E$ be such that $\left|z_{n}\right| \rightarrow \infty$ as $n \rightarrow \infty$ and for some $z_{0} \in \mathbb{Z}^{d} \times E$,

$$
\liminf _{n \rightarrow \infty} \frac{1}{\left|z_{n}\right|} \log \mathcal{G}\left(z_{0}, z_{n}\right) \geq 0 .
$$

Then

$$
\lim _{n \rightarrow \infty} \mathcal{G}\left(z+w, z_{n}\right) / \mathcal{G}\left(z+w^{\prime}, z_{n}\right)=1
$$

for all $z \in \mathbb{Z}^{d} \times E$ and for all those $w, w^{\prime} \in \mathbb{Z}^{d} \times\{0\}$ for which there is $n>0$ such that

$$
\inf _{z \in \mathbb{Z}^{d} \times E} \min \left\{p^{(n)}(z, z+w), p^{(n)}\left(z, z+w^{\prime}\right)\right\}>0
$$


This statement was initially obtained by Foley and McDonald 7 for Markov additive processes with an additive part on $\mathbb{Z}$ and for a sequence of the form $z_{n}=$ $(n, y)$ with a given $y \in E$. In general case, the proof of this proposition uses essentially the same ideas as in [7, we give this proof in section 8 .

Consider now the following more restrictive conditions.

(A1') (Communication condition) There exist $\theta>0$ and $C>0$ such that for any $z \neq z^{\prime}, z, z^{\prime} \in \mathbb{Z}^{d} \times E$ there is a sequence of points $z_{0}, z_{1}, \ldots, z_{n} \in \mathbb{Z}^{d} \times E$ with $z_{0}=z, z_{n}=z^{\prime}$ and $n \leq C\left|z^{\prime}-z\right|$ such that

$$
\left|z_{i}-z_{i-1}\right| \leq C \quad \text { and } \quad \mathbb{P}_{z_{i-1}}\left(\mathcal{Z}(1)=z_{i}\right) \geq \theta, \quad \forall i=1, \ldots, n .
$$

(A2') For every $z \in \mathbb{Z}^{d} \times E$, the function

$$
\varphi_{z}(a)=\mathbb{E}_{z}(\exp (a \cdot(\mathcal{Z}(1)-z)))
$$

is finite everywhere on $\mathbb{R}^{d+k}$ and the function $\hat{\varphi}$ defined by (3.3) is finite in a neighborhood of zero.

If the assumption (A1') is satisfied then there is a bounded function $n_{0}: E \rightarrow \mathbb{N}^{*}$ such that for any $z=(x, y) \in \mathbb{Z}^{d} \times E$,

$$
p^{\left(n_{0}(y)\right)}((x, y),(x, y)) \geq \theta^{n_{0}(y)}>0
$$

and hence, there is $k \in \mathbb{N}^{*}$ (for instance, $k=n$ ! with $n=\max _{y} n_{0}(y)$ ) such that

$$
p^{(k)}(z, z) \geq \theta^{k}, \quad \forall z \in \mathbb{Z}^{d} \times E .
$$

We denote by $\mathcal{K}$ the set of all integers $k>0$ for which

$$
\inf _{z \in Z^{d} \times E} p^{(k)}(z, z)>0
$$

The greatest common divisor of the set $\mathcal{K}$ is denoted by $\hat{k}$ and the following condition is assumed to be satisfied.

(A3) Up to multiplication by constants, there is a unique positive harmonic function $h: \mathbb{Z}^{d} \times E \rightarrow \mathbb{R}_{+}$satisfying the equality $h(z+\hat{k} w)=h(z)$ for all $z \in \mathbb{Z}^{d} \times E$ and $w \in \mathbb{Z}^{d} \times\{0\}$.

When the Markov-additive process $\mathcal{Z}(t)=(A(t), M(t))$ is stochastic, the last assumption means that the only positive harmonic functions $h: \mathbb{Z}^{d} \times E \rightarrow \mathbb{R}_{+}$ satisfying the equality $h(z+\hat{k} w)=h(z)$ for all $z \in \mathbb{Z}^{d} \times E$ and $w \in \mathbb{Z}^{d} \times\{0\}$ are constant.

Under these additional assumptions, using Proposition 3.2 we obtain the following statement.

Proposition 3.3. Suppose that the Markov-additive process $\mathcal{Z}(t)=(A(t), M(t))$ is transient and satisfies the hypotheses (A1') and (A2), and let a sequence of points $z_{n} \in \mathbb{Z}^{d} \times E$ with $\lim _{n}\left|z_{n}\right|=\infty$ satisfy the inequality (3.4) for some $z_{0} \in \mathbb{Z}^{d} \times E$. Then

$$
\lim _{n \rightarrow \infty} \mathcal{G}\left(z+\hat{k} w, z_{n}\right) / \mathcal{G}\left(z, z_{n}\right)=1, \quad \forall z \in \mathbb{Z}^{d} \times E, w \in \mathbb{Z}^{d} \times\{0\} .
$$

Moreover, if the conditions (A2') and (A3) are also satisfied, then

$$
\lim _{n \rightarrow \infty} \mathcal{G}\left(z, z_{n}\right) / \mathcal{G}\left(z^{\prime}, z_{n}\right)=h(z) / h\left(z^{\prime}\right), \quad \forall z, z^{\prime} \in \mathbb{Z}^{d} \times E .
$$


Proof. Let us show that

$$
\lim _{n \rightarrow \infty} \mathcal{G}\left(z+k w, z_{n}\right) / \mathcal{G}\left(z, z_{n}\right)=1
$$

for all $k \in \mathcal{K}, z \in \mathbb{Z}^{d} \times E$ and $w \in \mathbb{Z}^{d} \times\{0\}$. Indeed, according to the definition of the set $\mathcal{K}$, if $k \in \mathcal{K}$ then there is $\varepsilon>0$ such that

$$
p^{(k)}(z, z) \geq \varepsilon, \quad \forall z \in \mathbb{Z}^{d} \times E .
$$

Furthermore, because of the assumption (A1'), for every $w \in \mathbb{Z}^{d} \times\{0\}$, there is a bounded positive function $n_{w}(\cdot): E \rightarrow \mathbb{N}^{*}$ with $n_{w} \doteq \sup _{y \in E} n_{w}(y) \leq C|w|$ such that for any $(x, y) \in \mathbb{Z}^{d} \times E$,

$$
p^{\left(n_{w}(y)\right)}((x, y),(x, y)+w)=p^{\left(n_{w}(y)\right)}((0, y),(0, y)+w) \geq \theta^{n_{w}(y)} \geq \theta^{n_{w}} .
$$

From this it follows that

$$
p^{n_{w}(y)}((x, y)+(j-1) w,(x, y)+j w) \geq \theta^{n_{w}}
$$

for all $(x, y) \in \mathbb{Z}^{d} \times E, w \in \mathbb{Z}^{d} \times\{0\}$ and $j \in \mathbb{N}^{*}$ (to get the last inequality we replace in the above inequality $(x, y)$ by $(x, y)+(j-1) w)$. Using the inequalities (3.9) and (3.10) we get

$$
\begin{aligned}
p^{\left(k n_{w}\right)}(z, z+k w) & \geq\left(p^{(k)}(z, z)\right)^{n_{w}-n_{w}(y)} \prod_{j=1}^{k} p^{n_{w}(y)}(z+(j-1) w, z+j w) \\
& \geq \varepsilon^{n_{w}-n_{w}(y)} \theta^{k n_{w}} \geq \varepsilon^{n_{w}} \theta^{k n_{w}}
\end{aligned}
$$

for all $(x, y) \in \mathbb{Z}^{d} \times E, w \in \mathbb{Z}^{d} \times\{0\}$ and consequently,

$$
\inf _{z \in \mathbb{Z}^{d} \times E} \min \left\{p^{\left(k n_{w}\right)}(z, z), p^{\left(k n_{w}\right)}(z, z+\hat{k} w)\right\} \geq \min \left\{\varepsilon^{n_{w}}, \varepsilon^{n_{w}} \theta^{k n_{w}}\right\}>0
$$

for every $w \in \mathbb{Z}^{d} \times\{0\}$. By Proposition 3.2, under the hypotheses (A1') and (A2), the last inequality proves (3.8).

Consider now the subgroup $\langle\mathcal{K}\rangle$ of $\mathbb{Z}$ generated by the set $\mathcal{K}$ and let us notice that for every $k \in \mathcal{K}$, we have also

$$
\lim _{n \rightarrow \infty} \mathcal{G}\left(z-k w, z_{n}\right) / \mathcal{G}\left(z, z_{n}\right)=1, \quad \forall z \in \mathbb{Z}^{d} \times E, w \in \mathbb{Z}^{d} \times\{0\}
$$

To get this relation it is sufficient to replace $z$ by $z-k w$ in (3.8). Hence, (3.8) holds for every $k \in\langle\mathcal{K}\rangle$ and in particular for $k=\hat{k}$ because $\hat{k} \in\langle\mathcal{K}\rangle$ (see Lemma A.1 of Seneta [22]). The first assertion of Proposition 3.3 is therefore proved.

Suppose now that the conditions (A2') and (A3) are also satisfied. Because of the assumption $\left(\mathrm{A} 1^{\prime}\right)$, for any $z, z^{\prime} \in \mathbb{Z}^{d} \times E$, the probability that the Markov process $\mathcal{Z}(t)$ starting at $z$ ever hits $z^{\prime}$ is greater than $\theta^{C\left|z^{\prime}-z\right|}$ which implies that

$$
\theta^{C\left|z-z_{0}\right|} \leq \mathcal{G}\left(z, z_{n}\right) / \mathcal{G}\left(z_{0}, z_{n}\right) \leq \theta^{-C\left|z-z_{0}\right|}
$$

for all $z \in \mathbb{Z}^{d} \times E$. Suppose now that for a subsequence $\left(z_{n_{k}}\right)$, the sequence of functions

$$
\mathcal{G}\left(z, z_{n_{k}}\right) / \mathcal{G}\left(z_{0}, z_{n_{k}}\right), \quad z \in \mathbb{Z}^{d} \times E
$$

converge pointwise in $\mathbb{Z}^{d} \times E$. Then from the first inequality of (3.11) it follows that the function

$$
\tilde{h}(z) \doteq \lim _{k \rightarrow \infty} \mathcal{G}\left(z, z_{n_{k}}\right) / \mathcal{G}\left(z_{0}, z_{n_{k}}\right)
$$


is strictly positive. By dominated convergence theorem, the second inequality of (3.11) implies that the function $\tilde{h}$ is harmonic for $(\mathcal{Z}(t)$ ) (recall that under the hypotheses (A2'), the exponential functions are integrable with respect to the probability measure $p(z, \cdot)$ for every $z \in \mathbb{Z}^{d} \times E$ ). Moreover, the equality (3.6) shows that $\tilde{h}(z)=\tilde{h}(z+\hat{k} w)$ for all $z \in \mathbb{Z}^{d} \times E$ and $w \in \mathbb{Z}^{d} \times\{0\}$. Since the constant multiples of the function $h$ are the only harmonic functions satisfying this equality, we conclude that $\tilde{h}=c h$ with some $c>0$. Moreover, since $\tilde{h}\left(z_{0}\right)=1$ then $\tilde{h}(z)=h(z) / h\left(z_{0}\right)$ and consequently,

$$
\lim _{k \rightarrow \infty} \mathcal{G}\left(z, z_{n_{k}}\right) / \mathcal{G}\left(z_{0}, z_{n_{k}}\right)=\tilde{h}(z)=h(z) / h\left(z_{0}\right), \quad \forall z \in \mathbb{Z}^{d} \times E
$$

for every subsequence $n_{k}$ for which these limits exist. From this it follows that

$$
\lim _{n \rightarrow \infty} \mathcal{G}\left(z, z_{n}\right) / \mathcal{G}\left(z_{0}, z_{n}\right)=\tilde{h}(z)=h(z) / h\left(z_{0}\right), \quad \forall z \in \mathbb{Z}^{d} \times E
$$

because for every $z \in \mathbb{Z}^{d} \times E$, the sequence $\mathcal{G}\left(z, z_{n}\right) / \mathcal{G}\left(z_{0}, z_{n}\right)$ is bounded.

\section{SAMPle PATH LARGE DEVIATIONS}

4.1. General statement of SPLD principle. Let $D\left([0, T], \mathbb{R}^{d}\right)$ denote the set of all right continuous functions with left limits from $[0, T]$ to $\mathbb{R}^{d}$ endowed with Skorohod metric (see Billingsley [2]).

Definition 4.1. 1) A mapping $I_{[0, T]}: D\left([0, T], \mathbb{R}^{d}\right) \rightarrow[0,+\infty]$ is a good rate function on $D\left([0, T], \mathbb{R}^{d}\right)$ if for any $c \geq 0$ and any compact set $V \subset \mathbb{R}^{d}$, the set

$$
\left\{\phi \in D\left([0, T], \mathbb{R}^{d}\right): \phi(0) \in V \text { and } I_{[0, T]}(\phi) \leq c\right\}
$$

is compact in $D\left([0, T], \mathbb{R}^{d}\right)$. According to this definition, a good rate function is lower semi-continuous.

2) Let $(Z(t))$ be a Markov process on $E \subset \mathbb{Z}^{d}$ and let $Z^{\varepsilon}(t)=\varepsilon Z([t / \varepsilon])$ for $\varepsilon>0$. When $\varepsilon \rightarrow 0$, the family of scaled processes $\left(Z^{\varepsilon}(t)=\varepsilon Z([t / \varepsilon]), t \in[0, T]\right)$, is said to satisfy sample path large deviation principle in $D\left([0, T], \mathbb{R}^{d}\right)$ with a rate function $I_{[0, T]}$ if for any $z \in \mathbb{R}^{d}$

$$
\lim _{\delta \rightarrow 0} \liminf _{\varepsilon \rightarrow 0} \inf _{z^{\prime} \in \varepsilon E:\left|z^{\prime}-z\right|<\delta} \varepsilon \log \mathbb{P}_{\left[z^{\prime} / \varepsilon\right]}\left(Z^{\varepsilon}(\cdot) \in \mathcal{O}\right) \geq-\inf _{\phi \in \mathcal{O}: \phi(0)=z} I_{[0, T]}(\phi),
$$

for every open set $\mathcal{O} \subset D\left([0, T], \mathbb{R}^{d}\right)$, and

$$
\lim _{\delta \rightarrow 0} \limsup _{\varepsilon \rightarrow 0} \sup _{z^{\prime} \in \varepsilon E:\left|z^{\prime}-z\right|<\delta} \varepsilon \log \mathbb{P}_{\left[z^{\prime} / \varepsilon\right]}\left(Z^{\varepsilon}(\cdot) \in F\right) \leq-\inf _{\phi \in F: \phi(0)=z} I_{[0, T]}(\phi) .
$$

for every closed set $F \subset D\left([0, T], \mathbb{R}^{d}\right)$.

$\mathbb{P}_{[z / \varepsilon]}$ denotes here and throughout the distribution of the Markov process $(Z(t))$ corresponding to the initial state $Z(0)=[z / \varepsilon]$ where $[z / \varepsilon]$ is the nearest lattice point to $z / \varepsilon$ in $E \subset \mathbb{Z}^{d}$. We refer to sample path large deviation principle as SPLD principle. Inequalities (4.1) and (4.2) are referred as lower and upper SPLD bounds respectively. 
4.2. SPLD properties of scaled processes $Z_{+}^{\varepsilon}(t)=\varepsilon Z_{+}([t / \varepsilon])$. Under the hypotheses (A), the jump generating function $\varphi$ of (1.1) is finite everywhere on $\mathbb{R}^{d}$ and hence, by Mogulskii's theorem (see [6]), the family of scaled processes $Z^{\varepsilon}(t)=\varepsilon Z([t / \varepsilon])$ satisfies SPLD principle in $D\left([0, T], \mathbb{R}^{d}\right)$ with a good rate function

$$
I_{[0, T]}(\phi)= \begin{cases}\int_{0}^{T}(\log \varphi)^{*}(\dot{\phi}(t)) d t, & \text { if } \phi \text { is absolutely continuous, } \\ +\infty & \text { otherwise. }\end{cases}
$$

Recall that a continuous function $\phi:[0, T] \rightarrow \mathbb{R}^{d}$ is absolutely continuous if and only if it is differentiable almost everywhere in $[0, T]$ with $\dot{\phi} \in L^{1}([0, T])$ and for every $t \in[0, T]$,

$$
\phi(t)=\phi(0)+\int_{0}^{t} \dot{\phi}(s) d s
$$

(see Rudin 21]). The convex conjugate $(\log \varphi)^{*}$ of the function $\log \varphi$ is defined by

$$
(\log \varphi)^{*}(v) \doteq \sup _{a \in \mathbb{R}^{d}}(a \cdot v-\log \varphi(a)) .
$$

Under the hypotheses (A), $(\log \varphi)^{*}(v)=a \cdot v-\log \varphi(a)$ whenever $v=\nabla \varphi(a)$ because the function $(\log \varphi)$ is convex and differentiable everywhere in $\mathbb{R}^{d}$ (see Lemma 2.2.31 of the book of Dembo and Zeitouni [6]). In particular, according to the definition of the function $q \rightarrow a(q)$,

$$
(\log \varphi)^{*}(\nabla \varphi(a(q)))=a(q) \cdot \nabla \varphi(a(q))=|\nabla \varphi(a(q))| a(q) \cdot q
$$

because $\varphi(a(q))=1$. For a linear function $\phi(t)=v t$ with $v=\nabla \varphi(a(q))$ and for $T=1 /|\nabla \varphi(a(q))|$ we have therefore $\phi(T)=q$ and $I_{[0, T]}(\phi)=a \cdot q$.

To prove SPLD principle for the family of scaled processes $Z_{+}^{\varepsilon}(t)=\varepsilon Z_{+}([t / \varepsilon])$ we use the following lemma.

Lemma 4.1. If the random walk $(Z(t))$ is irreducible then the Markov process $\left(Z_{+}(t)\right)$ satisfies the communication condition $\left(A 1^{\prime}\right)$ : there are $\theta>0$ and $C>0$ such that for any $z \neq z^{\prime}, z, z^{\prime} \in \mathbb{Z}^{d-1} \times \mathbb{N}^{*}$ there exists a sequence of points $z_{0}, z_{1}, \ldots, z_{n} \in \mathbb{Z}^{d-1} \times \mathbb{N}^{*}$ with $z_{0}=z, z_{n}=z^{\prime}$ and $n \leq C\left|z^{\prime}-z\right|$ such that

$$
\left|z_{i}-z_{i-1}\right| \leq C \quad \text { and } \quad \mathbb{P}_{z_{i-1}}\left(Z_{+}(1)=z_{i}\right)=\mu\left(z_{i}-z_{i-1}\right) \geq \theta, \quad \forall i=1, \ldots, n .
$$

Proof. Indeed, suppose that the random walk $(Z(t))$ is irreducible. Then for every unit vector $e \in \mathbb{Z}^{d}$, there is a sequence of points $u_{1}^{e}, \ldots, u_{n_{e}}^{e} \in \mathbb{Z}^{d}$ such that $\mu\left(u_{i}^{e}\right)>0$ for every $i=1, \ldots, n_{e}$ and

$$
u_{1}^{e}+\ldots+u_{n_{e}}^{e}=e .
$$

For every $z \in \mathbb{Z}^{d}$ there are therefore $u_{1}, \ldots, u_{n} \in \cup_{e \in \mathbb{Z}^{d}:|e|=1}\left\{u_{1}^{e}, \ldots, u_{n_{e}}^{e}\right\}$ with

$$
n \leq \sum_{e \in \mathbb{Z}^{d}:|e|=1} n_{e} z \cdot e
$$

such that $z=u_{1}+\ldots+u_{n}$. This proves that the Markov process $(Z(t))$ satisfies the communication condition (A1') with

$$
\theta=\min _{e \in \mathbb{Z}^{d}:|e|=1} \min _{1 \leq i \leq n_{e}} \mu\left(u_{i}^{e}\right)
$$


and

$$
C=\max \left\{\sum_{e \in \mathbb{Z}^{d}:|e|=1} n_{e}, \max _{e \in \mathbb{Z}^{d}:|e|=1} \max _{1 \leq i \leq n_{e}}\left|u_{i}^{e}\right|\right\} .
$$

Hence, for any $z, z^{\prime} \in \mathbb{Z}^{d-1} \times \mathbb{N}^{*}$, if $z \neq z^{\prime}$ then there is a sequence of points $z_{0}, z_{1}, \ldots, z_{n} \in \mathbb{Z}^{d}$ with $z_{0}=z$ and $z_{n}=z^{\prime}$ such that $n \leq C\left|z^{\prime}-z\right|$,

$$
\left|z_{i}-z_{i-1}\right| \leq C \quad \text { and } \quad \mu\left(z_{i}-z_{i-1}\right) \geq \theta>0 \quad \forall i=1, \ldots, n .
$$

Moreover, without any restriction of generality we can suppose that there is $1 \leq k \leq$ $n$ such that $u_{i} \doteq z_{i}-z_{i-1} \in \mathbb{Z}^{d-1} \times \mathbb{N}$ for all $i<k$ and $u_{i} \doteq z_{i}-z_{i-1} \in \mathbb{Z}^{d-1} \times(-\mathbb{N})$ for $i>k$. Then, $z_{i} \doteq z+u_{1}+\ldots+u_{i} \in \mathbb{Z}^{d-1} \times \mathbb{N}^{*}$ for all $i=1, \ldots, n$ and consequently

$$
\mathbb{P}_{z_{i-1}}\left(Z_{+}(1)=z_{i}\right)=\mu\left(z_{i}-z_{i-1}\right) \geq \theta, \quad \forall i=1, \ldots, n .
$$

Proposition 4.1. If the random walk $(Z(t))$ is irreducible and the jump generating function $\varphi$ of (1.1) is finite everywhere on $\mathbb{R}^{d}$ then the sequence of scaled processes $Z_{+}^{\varepsilon}(t)=\varepsilon Z_{+}([t / \varepsilon])$ satisfies $S P L D$ principle in $D\left([0, T], \mathbb{R}^{d}\right)$ with the good rate function

$$
I_{[0, T]}^{+}(\phi)= \begin{cases}\int_{0}^{T}(\log \varphi)^{*}(\dot{\phi}(t)) d t, & \text { if } \phi \text { is absolutely continuous and } \\ & \phi(t) \in \mathbb{R}^{d-1} \times \mathbb{R}_{+} \text {for all } t \in[0, T], \\ +\infty & \text { otherwise. }\end{cases}
$$

Proof. Indeed, for any $c \geq 0$ and for any compact set $V \subset \mathbb{R}^{d}$, the set

$$
\begin{aligned}
\left\{\phi: I_{[0, T]}^{+}(\phi) \leq c, \phi(0) \in V\right\}=\left\{\phi: I_{[0, T]}(\phi)\right. & \leq c, \phi(0) \\
\{\phi: \phi(t) & \left.\in \mathbb{R}^{d-1} \times \mathbb{R}_{+}, \forall t \in[0, T]\right\} \\
\{\phi &
\end{aligned}
$$

is compact in $D\left([0, T], \mathbb{R}^{d}\right)$ because $I_{[0, T]}$ is a good rate function on $D\left([0, T], \mathbb{R}^{d}\right)$ and the set $\left\{\phi: \phi(t) \in \mathbb{R}^{d-1} \times \mathbb{R}_{+}, \forall t \in[0, T]\right\}$ is closed in $D\left([0, T], \mathbb{R}^{d}\right)$. This proves that the mapping $I_{[0, T]}^{+}: D\left([0, T], \mathbb{R}^{d}\right) \rightarrow[0,+\infty]$ is a good rate function.

Furthermore, for any closed set $F \subset D\left([0, T], \mathbb{R}^{d}\right)$, the set $F_{+} \doteq\{\phi \in F: \phi(t) \in$ $\left.\mathbb{R}^{d-1} \times \mathbb{R}_{+}, \forall t \in[0, T]\right\}$ is also closed and consequently, for any $z \in \mathbb{R}^{d-1} \times \mathbb{R}_{+}$, using Mogulskii's theorem we obtain

$$
\begin{aligned}
\lim _{\delta \rightarrow 0} \limsup _{\varepsilon \rightarrow 0} \sup _{z^{\prime}:\left|z^{\prime}-z\right|<\delta} \varepsilon & \log \mathbb{P}_{\left[z^{\prime} / \varepsilon\right]}\left(Z_{+}^{\varepsilon}(\cdot) \in F\right) \\
& =\lim _{\delta \rightarrow 0} \limsup _{\varepsilon \rightarrow 0} \sup _{z^{\prime}:\left|z^{\prime}-z\right|<\delta} \varepsilon \log \mathbb{P}_{\left[z^{\prime} / \varepsilon\right]}\left(Z_{+}^{\varepsilon}(\cdot) \in F_{+}\right) \\
& \leq \lim _{\delta \rightarrow 0} \limsup _{\varepsilon \rightarrow 0} \sup _{z^{\prime}:\left|z^{\prime}-z\right|<\delta} \varepsilon \log \mathbb{P}_{\left[z^{\prime} / \varepsilon\right]}\left(Z^{\varepsilon}(\cdot) \in F_{+}\right) \\
& \leq-\inf _{\phi \in F_{+}: \phi(0)=z} I_{[0, T]}(\phi)=-\inf _{\phi \in F: \phi(0)=z} I_{[0, T]}^{+}(\phi) .
\end{aligned}
$$

This proves that the sequence of rescaled processes $\left(Z_{+}^{\varepsilon}(t)\right)$ satisfies SPLD upper bound with the rate function $I_{[0, T]}^{+}$.

To get the SPLD lower bound it is sufficient to show that

$$
\lim _{\delta^{\prime} \rightarrow 0} \lim _{\delta \rightarrow 0} \liminf _{\varepsilon \rightarrow 0} \inf _{z:|z-\phi(0)|<\delta} \varepsilon \log \mathbb{P}_{[z / \varepsilon]}\left(\sup _{t \in[0, T]}\left|Z_{+}^{\varepsilon}(t)-\phi(t)\right|<\delta^{\prime}\right) \geq-I_{[0, T]}(\phi)
$$


for every absolutely continuous function $\phi:[0, T] \rightarrow \mathbb{R}^{d-1} \times \mathbb{R}_{+}$. If $\phi(t)$ belongs to the interior of the half-space $\mathbb{R}^{d-1} \times \mathbb{R}_{+}$for every $t \in[0, T]$, then this inequality follows from Mogulskii's theorem because in this case, for $\delta^{\prime}>0$ small enough, the process $(Z(t))$ does not exit from $\mathbb{Z}^{d-1} \times \mathbb{N}^{*}$ before the time $T / \varepsilon$ and $Z_{+}^{\varepsilon}(t)=Z^{\varepsilon}(t)$ for all $t \leq T$ whenever

$$
\sup _{t \in[0, T]}\left|Z^{\varepsilon}(t)-\phi(t)\right|<\delta^{\prime}
$$

Let now $\phi:[0, T] \rightarrow \mathbb{R}^{d-1} \times \mathbb{R}_{+}$be an arbitrary absolutely continuous function. For $\sigma>0$, consider the function $\phi_{\sigma}$ on $[0, T]$ defined by

$$
\phi_{\sigma}(t)=\phi(t)+\sigma e_{d}
$$

where $e_{d}=(0, \ldots, 0,1) \in \mathbb{Z}^{d}$. Because of Lemma 4.1 there are $C \geq 1$ and $\theta>0$ such that for any $z \in \mathbb{R}^{d}$ satisfying the inequality $|\phi(0)-z|<\delta$ and for any $\varepsilon>0$ there is a sequence of points $z_{0}, z_{1}, \ldots, z_{N} \in \mathbb{Z}^{d-1} \times \mathbb{N}^{*}$ with $z_{0}=[z / \varepsilon]$ and $z_{N}=\left[\phi_{\sigma}(0) / \varepsilon\right]$ for which the following inequalities hold

$$
\begin{aligned}
N & \leq C\left|[z / \varepsilon]-\left[\phi_{\sigma}(0) / \varepsilon\right]\right| \leq 2 d C+\left|z-\phi_{\sigma}(0)\right| / \varepsilon \\
& \leq 2 d C+(\delta+\sigma) / \varepsilon \\
\left|z_{i}-z_{i-1}\right| & \leq C \text { and } p\left(z_{i-1}, z_{i}\right) \geq \theta, \quad \forall i=1, \ldots, N .
\end{aligned}
$$

Hence, on the event

$E_{\sigma, \sigma^{\prime}, \varepsilon}=\left\{Z_{+}(0)=z_{0}, \ldots, Z_{+}(N)=z_{N}\right\} \cap\left\{\sup _{t \in[0, T]}\left|Z_{+}^{\varepsilon}(t+N \varepsilon)-\phi_{\sigma}(t)\right|<\sigma^{\prime}\right\}$,

for $t \in[0, N \varepsilon]$, using the inequality (4.5) and the first inequalit of (4.6) we obtain

$$
\begin{aligned}
\left|Z_{+}^{\varepsilon}(t)-\phi(t)\right| & \leq\left|Z_{+}^{\varepsilon}(t)-\phi_{\sigma}(0)\right|+\left|\phi_{\sigma}(0)-\phi(0)\right|+|\phi(0)-\phi(t)| \\
& \leq\left|Z_{+}^{\varepsilon}(t)-\phi_{\sigma}(0)\right|+\sigma+\max _{t \in[0, N \varepsilon]}|\phi(0)-\phi(t)| \\
& \leq \max _{i=1, \ldots, N}\left|\varepsilon z_{i}-\phi_{\sigma}(0)\right|+\sigma+\max _{t \in[0, N \varepsilon]}|\phi(0)-\phi(t)| \\
& \leq \varepsilon \max _{i=1, \ldots, N}\left|z_{i}-z_{N}\right|+\varepsilon d+\sigma+\max _{t \in[0, N \varepsilon]}|\phi(0)-\phi(t)| \\
& \leq \varepsilon(N C+d)+\sigma+\max _{t \in[0, N \varepsilon]}|\phi(0)-\phi(t)| \\
& \leq 2 d C^{2} \varepsilon+(\delta+\sigma) C+\varepsilon d+\sigma+\max _{\substack{s, s^{\prime} \in[0, T]: \\
\left|s^{\prime}-s\right| \leq \delta+\sigma+2 d C \varepsilon}}\left|\phi(s)-\phi\left(s^{\prime}\right)\right|
\end{aligned}
$$

and for $t \in[N \varepsilon, T]$ we get

$$
\begin{aligned}
\left|Z_{+}^{\varepsilon}(t)-\phi(t)\right| & \leq\left|Z_{+}^{\varepsilon}(t)-\phi_{\sigma}(t-N \varepsilon)\right|+\left|\phi_{\sigma}(t-N \varepsilon)-\phi(t)\right| \\
& \leq \sigma^{\prime}+\left|\phi_{\sigma}(t-N \varepsilon)-\phi(t)\right| \\
& \leq \sigma^{\prime}+\sigma+|\phi(t-N \varepsilon)-\phi(t)| \\
& \leq \sigma^{\prime}+\sigma+\max _{\substack{s, s^{\prime} \in[0, T]: \\
\left|s^{\prime}-s\right| \leq \delta+\sigma+2 d C \varepsilon}}\left|\phi(s)-\phi\left(s^{\prime}\right)\right|
\end{aligned}
$$

where

$$
\max _{\substack{s, s^{\prime} \in[0, T]: \\\left|s^{\prime}-s\right| \leq \delta+\sigma+2 d C \varepsilon}}\left|\phi(s)-\phi\left(s^{\prime}\right)\right| \rightarrow 0
$$


when $\delta, \sigma, \varepsilon \rightarrow 0$ because the function $\phi$ is continuous on $[0, T]$. These inequalities show that for any $\delta^{\prime}>0$ there is $\sigma_{0}>0$ such that for all $\left.\sigma, \delta, \sigma^{\prime}, \varepsilon \in\right] 0, \sigma_{0}[$, on the event $E_{\sigma, \sigma^{\prime}, \varepsilon}$ the following inequality holds

$$
\sup _{t \in[0, T]}\left|Z_{+}^{\varepsilon}(t)-\phi(t)\right|<\delta^{\prime}
$$

Using the second inequality of (4.6) and Markov property from this it follows that for all $0<\varepsilon, \delta, \sigma^{\prime}<\sigma<\sigma_{0}$,

$$
\begin{aligned}
\varepsilon \log \mathbb{P}_{[z / \varepsilon]}\left(\sup _{t \in[0, T]}\left|Z_{+}^{\varepsilon}(t)-\phi(t)\right|<\delta^{\prime}\right) \\
\quad \geq N \varepsilon \log \theta+\varepsilon \log \mathbb{P}_{\left[\phi_{\sigma}(0) / \varepsilon\right]}\left(\sup _{t \in[0, T]}\left|Z_{+}^{\varepsilon}(t)-\phi_{\sigma}(t)\right|<\sigma^{\prime}\right) \\
\geq(2 d C \varepsilon+\delta+\sigma) \log \theta+\varepsilon \log \mathbb{P}_{\left[\phi_{\sigma}(0) / \varepsilon\right]}\left(\sup _{t \in[0, T]}\left|Z_{+}^{\varepsilon}(t)-\phi_{\sigma}(t)\right|<\sigma^{\prime}\right)
\end{aligned}
$$

and consequently,

$$
\begin{aligned}
& \liminf _{\varepsilon \rightarrow 0} \varepsilon \log \mathbb{P}_{[z / \varepsilon]}\left(\sup _{t \in[0, T]}\left|Z_{+}^{\varepsilon}(t)-\phi(t)\right|<\delta^{\prime}\right) \\
& \geq(\delta+\sigma) \log \theta+\liminf _{\varepsilon \rightarrow 0} \varepsilon \log \mathbb{P}_{\left[\phi_{\sigma}(0) / \varepsilon\right]}\left(\sup _{t \in[0, T]}\left|Z_{+}^{\varepsilon}(t)-\phi_{\sigma}(t)\right|<\sigma^{\prime}\right) \\
&(4.7) \quad \geq(\delta+\sigma) \log \theta+\liminf _{\varepsilon \rightarrow 0} \varepsilon \log \mathbb{P}_{\left[\phi_{\sigma}(0) / \varepsilon\right]}\left(\sup _{t \in[0, T]}\left|Z^{\varepsilon}(t)-\phi_{\sigma}(t)\right|<\sigma^{\prime}\right)
\end{aligned}
$$

where the last inequality holds because for $0<\sigma^{\prime}<\sigma$, on the event

$$
\left\{\sup _{t \in[0, T]}\left|Z^{\varepsilon}(t)-\phi_{\sigma}(t)\right|<\sigma^{\prime}\right\},
$$

we have the equality $Z_{+}(t)=Z(t)$ for all $t \in[0, T]$. Relation (4.7) combined with SPLD lower bound of Mogulskii's theorem shows that the left hand side of (4.4) is greater than

$\limsup _{\sigma \rightarrow 0} \lim _{\sigma^{\prime} \rightarrow 0} \liminf _{\varepsilon \rightarrow 0} \varepsilon \log \mathbb{P}_{\left[\phi_{\sigma}(0) / \varepsilon\right]}\left(\sup _{t \in[0, T]}\left|Z^{\varepsilon}(t)-\phi_{\sigma}(t)\right|<\sigma^{\prime}\right) \geq-\liminf _{\sigma \rightarrow 0} I_{[0, T]}\left(\phi_{\sigma}\right)$ and hence, using the equalities

$$
I_{[0, T]}\left(\phi_{\sigma}\right)=\int_{0}^{T}(\log \varphi)^{*}\left(\dot{\phi}_{\sigma}(t)\right) d t=\int_{0}^{T}(\log \varphi)^{*}(\dot{\phi}(t)) d t=I_{[0, T]}(\phi)
$$

we obtain (4.4)

4.3. Large deviation estimates for the Green's functions. The lower rough logarithmic estimates for the Green's function $G_{+}\left(z, z^{\prime}\right)$ are derived now from the SPLD properties of the scaled processes $Z_{+}^{\varepsilon}(t)=\varepsilon Z_{+}([t / \varepsilon])$.

Proposition 4.2. Under the hypotheses (A), for any $q \in \mathcal{S}_{+}^{d}$ and $z \in \mathbb{Z}^{d-1} \times \mathbb{N}^{*}$,

$$
\liminf _{n \rightarrow \infty} \frac{1}{\left|z_{n}\right|} \log G_{+}\left(z, z_{n}\right) \geq-a(q) \cdot q
$$


when $\left|z_{n}\right| \rightarrow \infty$ and $z_{n} /\left|z_{n}\right| \rightarrow q$ as $n \rightarrow \infty$, with $z_{n} \in \mathbb{Z}^{d-1} \times \mathbb{N}^{*}$.

Proof. Indeed, if the hypotheses (A) are satisfied then by Lemma 4.1, the Markov process $\left(Z_{+}(t)\right)$ satisfies communication condition (A1') and hence, for any $z, z^{\prime} \in$ $\mathbb{Z}^{d-1} \times \mathbb{N}^{*}$, if $z \neq z^{\prime}$ then there is $0<t \leq C\left|z-z^{\prime}\right|$ such that

$$
\mathbb{P}_{z}\left(Z_{+}(t)=z^{\prime}\right) \geq \theta^{t} \geq \theta^{C\left|z^{\prime}-z\right|} .
$$

Using the inequality $G_{+}\left(z, z_{n}\right) \geq G_{+}\left(z, z^{\prime}\right) \mathbb{P}_{z^{\prime}}\left(Z_{+}(t)=z_{n}\right)$ for $z^{\prime} \neq z_{n}$, we get therefore

$$
G_{+}\left(z, z_{n}\right) \geq G_{+}\left(z, z^{\prime}\right) \theta^{C\left|z^{\prime}-z_{n}\right|}, \quad \forall n \in \mathbb{N}, z^{\prime} \in \mathbb{Z}^{d-1} \times \mathbb{N}^{*} .
$$

Using moreover the inequality

$$
\operatorname{Card}\left\{z \in \mathbb{Z}^{d}:|z-a|<\delta R\right\} \leq(2 \delta R+1)^{d}
$$

with $R=\left|z_{n}\right|$ and $a=\left|z_{n}\right| q$ we obtain

$$
G_{+}\left(z, z_{n}\right) \geq \frac{1}{\left(2 \delta\left|z_{n}\right|+1\right)^{d}} \theta^{2 C \delta\left|z_{n}\right|} \sum_{z^{\prime}:\left|q-z^{\prime} /\right| z_{n}||<\delta} G_{+}\left(z, z^{\prime}\right)
$$

for all those $n \in \mathbb{N}$ for which $\left|q-z_{n} /\right| z_{n}||<\delta$ and consequently,

$$
\begin{aligned}
\lim _{n \rightarrow \infty} \frac{1}{\left|z_{n}\right|} \log G_{+}\left(z, z_{n}\right) & \geq-2 C \delta \log \theta+\liminf _{n \rightarrow \infty} \frac{1}{\left|z_{n}\right|} \log \sum_{z^{\prime}:\left|q-z^{\prime} /\right| z_{n}||<\delta} G_{+}\left(z, z^{\prime}\right) \\
& \geq-2 C \delta \log \theta+\liminf _{\varepsilon \rightarrow 0} \varepsilon \log \sum_{z^{\prime}:\left|q-\varepsilon z^{\prime}\right|<\delta} G_{+}\left(z, z^{\prime}\right) .
\end{aligned}
$$

Finally, letting $\delta \rightarrow 0$ and using SPLD lower bound for the family of scaled process $Z_{+}^{\varepsilon}(t)=\varepsilon Z_{+}([t / \varepsilon])$ we conclude that

$$
\begin{aligned}
\lim _{n \rightarrow \infty} \frac{1}{\left|z_{n}\right|} \log G_{+}\left(z, z_{n}\right) & \geq \lim _{\delta \rightarrow 0} \liminf _{\varepsilon \rightarrow 0} \varepsilon \log \sum_{z^{\prime}:\left|q-\varepsilon z^{\prime}\right|<\delta} G_{+}\left(z, z^{\prime}\right) \\
& \geq \lim _{\delta \rightarrow 0} \liminf _{\varepsilon \rightarrow 0} \varepsilon \log \mathbb{P}_{z}\left(\left|Z_{+}^{\varepsilon}(T)-q\right|<\delta\right) \\
& \geq-\inf _{\phi: \phi(0)=0, \phi(T)=q} I_{[0, T]}^{+}(\phi)
\end{aligned}
$$

for every $T>0$. To get (4.8) it is sufficient now to notice that the right hand side of the last inequality is greater than $-a(q) \cdot q$ because for $T=1 /|\nabla \varphi(a(q))|$ and for the linear function $\phi(t)=v t$ with $v=\nabla \varphi(a(q))=q|\nabla \varphi(a(q))|$, from (4.3) it follows that

$$
I_{[0, T]}^{+}(\phi)=T(\log \varphi)^{*}(v)=T a(q) \cdot v=a(q) \cdot q
$$

\section{HARMONIC FUNCTIONS}

The harmonic functions of the Markov process $\left(Z_{+}(t)\right)$ are now identified. The main result of this section is the following proposition. 
Proposition 5.1. Under the hypotheses (A), the following assertions hold. 1) A non-negative function $h$ is harmonic for the Markov process $\left(Z_{+}(t)\right)$ if and only if there is a positive measure $\nu_{h}$ on $\partial_{+} D$ such that

$$
h(z)=\int_{\partial_{+} D} h_{a,+}(z) d \nu_{h}(a), \quad \forall z \in \mathbb{N}^{*} \times \mathbb{Z}^{d-1} .
$$

2) For every $a=(\alpha, \beta) \in \partial_{+} D$ with $\alpha \in \mathbb{R}^{d-1}$ and $\beta \in \mathbb{R}$, the constant multiples of of the function $h_{a,+}$ are the only non-negative harmonic functions for which

$$
\sup _{x \in \mathbb{R}^{d-1}} \exp (-\alpha \cdot x) h(x, y)<+\infty, \quad \forall y \in \mathbb{N}^{*} .
$$

3) The constant multiples of the functions $h_{a,+}$ with $a \in \partial_{+} D$, are the only minimal harmonic functions of the Markov process $\left(Z_{+}(t)\right)$.

Throughout this section the following notations are used : for $a=(\alpha, \beta) \in \mathbb{R}^{d}$ we denote by $\alpha$ [resp. $\beta$ ] the vector of $d-1$ first coordinates [resp. last coordinate] of $a$. For $z=(x, y) \in \mathbb{Z}^{d}$ the variavles $x$ and $y$ are defined in the similar way.

To prove Proposition 5.1 we combine the properties of Markov-additive processes and the results of Doney [ 8 . The Markov process $\left(Z_{+}(t)\right)=\left(X_{+}(t), Y_{+}(t)\right)$ is Markov-additive with an additive part $X_{+}(t)$ taking the values in $Z^{d-1}$ and a Markovian part $Y_{+}(t)$ taking the values in $\mathbb{N}^{*}$. Under the hypotheses $(A)$, the Markovian part $Y_{+}(t)$ is irreducible on $\mathbb{N}^{*}$ and hence, the Feynman-Kac transfom matrix $\mathcal{P}_{+}(\alpha)=\left(\mathcal{P}_{+}\left(\alpha, y, y^{\prime}\right), y, y^{\prime} \in \mathbb{N}^{*}\right)$ with

$$
\mathcal{P}_{+}\left(\alpha, y, y^{\prime}\right)=\mathbb{E}_{(0, y)}\left(\exp \left(\alpha \cdot X_{+}(1)\right) ; Y_{+}(1)=y^{\prime}\right)
$$

is also irreducible. The quantity $e^{\lambda_{+}(\alpha)}$ with

$$
\lambda_{+}(\alpha) \doteq \limsup _{n} \frac{1}{n} \log \mathcal{P}_{+}^{(n)}\left(\alpha, y, y^{\prime}\right)
$$

is the spectral radius of the transform matrix $\mathcal{P}_{+}(\alpha)$. Recall that this limit does not depend on $y, y^{\prime} \in \mathbb{N}^{*}$ because the matrix $\mathcal{P}_{+}(\alpha)$ is irreducible (see [22]).

To identify the harmonic functions of the Markov process $\left(Z_{+}(t)\right)$ we identify first the function $\lambda_{+}(\cdot)$. This is a subject of the following lemma.

Lemma 5.1. Under the hypotheses $(A)$,

$$
\lambda_{+}(\alpha)=\inf _{\beta \in \mathbb{R}} \log \varphi(\alpha, \beta), \quad \forall \alpha \in \mathbb{R}^{d-1} .
$$

Proof. To prove this lemma we consider a random walk $(Z(t))=(X(t), Y(t))$ on $\mathbb{Z}^{d}$ having transition probabilities $p\left(z, z^{\prime}\right)=\mu\left(z^{\prime}-z\right)$ as a Markov-additive process on $\mathbb{Z}^{d-1} \times \mathbb{Z}$ with an additive part $(X(t))$ on $\mathbb{Z}^{d-1}$ and a Markovian part $(Y(t))$ on $Z$. For such a Markov-additive process, the Feynman-Kac transfom matrix $\mathcal{P}(\alpha)=\left(\mathcal{P}\left(\alpha, y, y^{\prime}\right), y, y^{\prime} \in \mathbb{Z}\right)$ is defined by

$$
\mathcal{P}\left(\alpha, y, y^{\prime}\right)=\mathbb{E}_{(0, y)}\left(\exp (\alpha \cdot X(1)) ; Y(1)=y^{\prime}\right), \quad \forall y, y \in \mathbb{Z}
$$

and its spectral radius $e^{\lambda(\alpha)}$ is given by

$$
\lambda(\alpha) \doteq \limsup _{t \rightarrow \infty} \frac{1}{t} \log \mathbb{P}_{(0, y)}\left(\exp (\alpha \cdot X(t)), Y(t)=y^{\prime}\right) .
$$

The first step of our proof shows that

$$
\lambda(\alpha)=\inf _{\beta \in \mathbb{R}} \log \varphi(\alpha, \beta) .
$$


Indeed, under the hypotheses $(A)$, the function $\varphi$ is convex and has compact level sets. For every $\alpha$, there is therefore $\beta_{\alpha}^{0} \in \mathbb{R}$ such that

$$
\inf _{\beta \in \mathbb{R}} \varphi(\alpha, \beta)=\varphi\left(\alpha, \beta_{\alpha}^{0}\right) .
$$

A twisted random walk $(\tilde{Y}(t))$ on $\mathbb{Z}$ with transition probabilities

$$
\tilde{p}\left(y, y^{\prime}\right)=\sum_{x \in \mathbb{Z}^{d-1}} \mu\left(x, y^{\prime}-y\right) \exp \left(\alpha \cdot x+\beta_{\alpha}^{0}\left(y^{\prime}-y\right)\right) / \varphi\left(\alpha, \beta_{\alpha}^{0}\right)
$$

has finite variance and zero mean because

$$
\mathbb{E}(\tilde{Y}(1))=\frac{\partial}{\partial \beta} \log \varphi\left(\alpha, \beta_{\alpha}^{0}\right)=0,
$$

from which it follows that

$$
\limsup _{t \rightarrow \infty} \frac{1}{t} \log \tilde{p}^{(t)}\left(y, y^{\prime}\right)=0
$$

for all $y, y^{\prime} \in \mathbb{Z}$ (see Spitzer [23]). The last relation combined with the equality

$$
\mathbb{E}_{(0, y)}\left(\exp (\alpha \cdot X(t)), Y(t)=y^{\prime}\right)=\left(\varphi\left(\alpha, \beta_{\alpha}^{0}\right)\right)^{t} \exp \left(\beta_{\alpha}^{0}\left(y-y^{\prime}\right)\right) \tilde{p}^{(t)}(y, y) .
$$

proves $(5.3)$.

Now, to complete the proof of Lemma 5.1, we show that $\lambda_{+}(\alpha)=\lambda(\alpha)$. For this we notice that $\mathcal{P}\left(\alpha ; y, y^{\prime}\right)=\mathcal{P}_{+}\left(\alpha ; y, y^{\prime}\right)$ for all $y, y^{\prime} \in \mathbb{N}^{*}$. Since under the Assumption $(A)$, the matrices $\mathcal{P}_{+}(\alpha)=\left(\mathcal{P}\left(\alpha ; y, y^{\prime}\right), y, y^{\prime} \in \mathbb{N}^{*}\right)$ and $\mathcal{P}(\alpha)=$ $\left(\mathcal{P}\left(\alpha ; y, y^{\prime}\right), y, y^{\prime} \in \mathbb{Z}\right)$ are irreducible then by Theorem 6.3 of Seneta 22. (see also Proposition 2 of Ignatiouk [13]),

$$
\lambda(\alpha)=\sup _{K \subset \subset \mathbb{Z}} \lambda_{K}(\alpha) \text { and } \lambda_{+}(\alpha)=\sup _{K \subset \subset \mathbb{N}^{*}} \lambda_{K}(\alpha)
$$

where both supremums are taken over all finite subsets $K$ and for any finite set $K \subset \mathbb{Z}, \exp \left(\lambda_{K}(\alpha)\right)$ is the maximal real eigenvalue of the truncated matrix $\left(\mathcal{P}\left(\alpha ; y, y^{\prime}\right) ; y, y^{\prime} \in K\right)$. Moreover, since $\mathcal{P}\left(\alpha ; y^{\prime}+y, y^{\prime \prime}+y\right)=\mathcal{P}\left(\alpha ; y^{\prime}, y^{\prime \prime}\right)$ for all $y, y^{\prime}, y^{\prime \prime} \in \mathbb{Z}$, then for every finite set $K \subset \mathbb{Z}$ we have also

$$
\lambda_{K}(\alpha)=\lambda_{K+y}(\alpha), \quad \forall y \in \mathbb{Z}
$$

and consequently, $\lambda_{+}(\alpha)=\lambda(\alpha)$.

Remark that by Lemma $5.1 \lambda_{+}(\alpha) \leq 0$ if and only if $\varphi(\alpha, \beta) \leq 1$ for some $\beta \in \mathbb{R}$ and hence, the mapping $a=(\alpha, \beta) \rightarrow \alpha$ determines a one to one and on correspondence from $\partial_{+} D$ to $\left\{\alpha \in \mathbb{R}^{d-1}: \lambda_{+}(\alpha) \leq 0\right\}$. Using Lemma 5.1 and Proposition 3.1 we obtain therefore the following statement.

Lemma 5.2. Under the Assumptions $(A)$, every minimal harmonic function $h$ of the Markov process $\left(Z_{+}(t)\right)$ is of the form

$$
h(x, y)=\exp (\alpha \cdot x) h(0, y)>0, \quad \forall(x, y) \in \mathbb{Z}^{d-1} \times \mathbb{N}^{*}
$$

for some $a=(\alpha, \beta) \in \partial_{+} D$.

To identify the harmonic functions satisfying the equation (5.4) we need the following lemma. 
Lemma 5.3. For every aperiodic random walk $(Y(t))$ on $\mathbb{Z}$ having transition probabilities $P\left(y, y^{\prime}\right)=P\left(0, y^{\prime}-y\right)$ such that for some $\delta<0$,

$$
\sum_{y} e^{-\delta y} P(0, y)<\infty, \quad \sum_{y}|y| P(0, y)<\infty, \quad \text { and } \quad m \doteq \sum_{y} y P(0, y) \geq 0,
$$

the constant multiples of the function

$$
f(y)= \begin{cases}\mathbb{P}_{y}(\tau=+\infty) & \text { if } m>0, \\ y-\mathbb{E}_{y}(Y(\tau)) & \text { if } m=0,\end{cases}
$$

with $\tau=\inf \{t \geq 0: Y(t) \leq 0\}$, are the only positive solutions of the equation

$$
\sum_{y^{\prime}>0} P\left(y, y^{\prime}\right) f\left(y^{\prime}\right)=f(y), \quad y \in \mathbb{N}^{*} .
$$

Proof. This statement follows from Theorem 1 of Doney $[$ ] (when $m=0$ this is a consequence of Example E 27.3 in Chapter VI of Spitzer [23]). This theorem proves that for an aperiodic random walk $(Y(t))$ on $\mathbb{Z}$ having transition probabilities $P\left(y, y^{\prime}\right)=P\left(0, y^{\prime}-y\right)$ and a non-negative mean $m \geq 0$, the only positive solutions of the equation

$$
\sum_{y^{\prime} \geq 0} P\left(y, y^{\prime}\right) f\left(y^{\prime}\right)=f(y), \quad y \in \mathbb{N} .
$$

are the constant multiples of the renewal function of strict increasing ladder heights of $(-Y(t))$. Hence, to prove Lemma 5.3 it is sufficient to show that the function (5.5) is well defined and satisfies the equation (5.6).

When $m \neq 0$ the function $f$ is clearly positive and well defined. Suppose now that $m=0$ and let us consider the function

$$
g_{+}\left(y, y^{\prime}\right)=\sum_{t=0}^{\infty} \mathbb{P}_{y}\left(Y(t)=y^{\prime}, \tau>t\right) .
$$

In this case, for any $\delta>0$,

$$
\begin{aligned}
0 \leq-\mathbb{E}_{y}(Y(\tau)) & \leq \frac{1}{\delta} \mathbb{E}_{y}\left(e^{-\delta Y(\tau)}\right)=\sum_{y^{\prime}>0} g_{+}\left(y, y^{\prime}\right) \sum_{y^{\prime \prime} \leq 0} e^{-\delta y^{\prime \prime}} P\left(y^{\prime}, y^{\prime \prime}\right) \\
& \leq \sum_{y^{\prime \prime}} e^{-\delta y^{\prime \prime}} P\left(0, y^{\prime \prime}\right) \sum_{y^{\prime}>0} g_{+}\left(y, y^{\prime}\right) e^{-\delta y^{\prime}} \\
& \leq \sum_{y^{\prime \prime}} e^{-\delta y^{\prime \prime}} P\left(0, y^{\prime \prime}\right) g_{+}(y, y) \sum_{y^{\prime}>0} e^{-\delta y^{\prime}}
\end{aligned}
$$

where the last inequality holds because for the "reversed" random walk $\tilde{Y}(t)$ with transition probabilities $\tilde{P}\left(y, y^{\prime}\right)=P\left(0, y-y^{\prime}\right)=P\left(y^{\prime}, y\right)$,

$$
\begin{aligned}
g_{+}\left(y, y^{\prime}\right) & =\sum_{t=0}^{\infty} \mathbb{P}_{y}\left(Y(t)=y^{\prime}, \tau>t\right)=\sum_{t=0}^{\infty} \mathbb{P}_{y^{\prime}}(\tilde{Y}(t)=y, \tau>t) \\
& \leq \mathbb{P}_{y^{\prime}}(\tilde{Y}(t)=y \text { for some } t \geq 0) \sum_{t=0}^{\infty} \mathbb{P}_{y}(\tilde{Y}(t)=y, \tau>t) \\
& \leq \sum_{t=0}^{\infty} \mathbb{P}_{y}(\tilde{Y}(t)=y, \tau>t)=g_{+}(y, y)
\end{aligned}
$$

for every $y^{\prime} \in \mathbb{N}^{*}$. This proves that the function $f$ is positive and finite. 
Finally, straightforward calculations show that the function $f$ satisfies the equa$\operatorname{tion}(5.6)$.

The harmonic functions satisfying the equation (5.4) are now identified.

Lemma 5.4. Under the Assumptions $(A)$, for every $a=(\alpha, \beta) \in \partial_{+} D$, the constant multiples of $h_{a,+}$ are the only harmonic functions of the Markov process $\left(Z_{+}(t)\right)$ for which (5.4) holds with a given $\alpha$.

Proof. To prove this lemma it is sufficient to show that for every $a \in \partial_{+} D$, the constant multiples of $\tilde{h}_{a,+}(z)=\exp (-a \cdot z) h_{a,+}(z)$ are the only positive solutions of the equation

$$
\sum_{z^{\prime} \in \mathbb{Z}^{d-1} \times \mathbb{N}^{*}} p\left(z, z^{\prime}\right) \exp \left(a \cdot\left(z^{\prime}-z\right)\right) \tilde{h}\left(z^{\prime}\right)=\tilde{h}(z), \quad z \in \mathbb{Z}^{d-1} \times \mathbb{N}^{*}
$$

satisfying the equality $\tilde{h}(x, y)=\tilde{h}(0, y)$ for all $(x, y) \in \mathbb{Z}^{d-1} \times \mathbb{N}^{*}$. To prove such a property for a given $a=(\alpha, \beta) \in \partial_{+} D$, we consider a twisted random walk $\tilde{Z}(t)=(\tilde{X}(t), \tilde{Y}(t))$ on $\mathbb{Z}^{d}$ with transition probabilities

$$
\tilde{p}\left(z, z^{\prime}\right)=\exp \left(a \cdot\left(z^{\prime}-z\right)\right) \mu\left(z^{\prime}-z\right) .
$$

Then for every $z=(x, y) \in \mathbb{Z}^{d-1} \times \mathbb{N}^{*}$,

$$
\tilde{h}_{a,+}(z)=f_{a}(y) \doteq \begin{cases}\mathbb{P}_{y}\left(\tau_{a}=+\infty\right) & \text { if } a \in \partial_{+} D \backslash \partial_{0} D \\ y-\mathbb{E}_{y}\left(\tilde{Y}\left(\tau_{a}\right)\right) & \text { if } a \in \partial_{0} D\end{cases}
$$

where $\tau_{a}=\inf \{t \geq 0: \tilde{Y}(t) \leq 0\}$. Moreover, because of the assumption (A), the last coordinate $(\tilde{Y}(t))$ of $(\tilde{Z}(t))$ is an aperiodic random walk on $\mathbb{Z}$ with transition probabilities

$$
P_{a}\left(y, y^{\prime}\right)=P_{a}\left(0, y^{\prime}-y\right)=\sum_{x \in \mathbb{Z}^{d-1}} \mu\left(x, y^{\prime}-y\right) \exp \left(\alpha \cdot x+\beta\left(y^{\prime}-y\right)\right)
$$

and mean

$$
m_{a} \doteq \mathbb{E}_{0}(\tilde{Y}(1))=\sum_{z=(x, y) \in \mathbb{Z}^{d}} y \mu(z) \exp (a \cdot z)=\frac{\partial}{\partial \beta} \varphi(\alpha, \beta) \geq 0
$$

where $m_{a}=0$ if and only if $a \in \partial_{0} D$. Hence, by Lemma 5.3. the constant multiples of the function $f_{a}$ are the only positive solution of the equation

$$
\sum_{y^{\prime}>0} P_{a}\left(y, y^{\prime}\right) f\left(y^{\prime}\right)=f(y), \quad y \in \mathbb{N}^{*}
$$

and therefore, the constant multiples of $\tilde{h}_{a,+}$ are the only positive solutions of the equation (5.7) satisfying the equality $\tilde{h}(x, y)=\tilde{h}(0, y)$ for all $(x, y) \in \mathbb{Z}^{d-1} \times \mathbb{N}^{*}$.

The last lemma combined with Lemma 5.2 proves the following statement.

Lemma 5.5. Under the hypotheses $(A)$, every minimal harmonic function of the Markov process $\left(Z_{+}(t)\right)$ is of the form $h=c h_{a,+}$ with some $c>0$ and $a \in \partial_{+} D$. 
Proof of Proposition 5.1. We are ready now to prove the representation (5.1). By the Poisson-Martin representation theorem (see Woess 24]), every non-negative harmonic function of the Markov process $\left(Z_{+}(t)\right)$ is of the form

$$
h(z)=\int_{\partial_{m}\left(\mathbb{Z}^{d-1} \times \mathbb{N}^{*}\right)} K_{+}(z, \gamma) d \tilde{\nu}_{h}(\gamma), \quad \forall z \in \mathbb{Z}^{d-1} \times \mathbb{N}^{*}
$$

with some Borel measure $\tilde{\nu}_{h} \geq 0$ on the minimal Martin boundary $\partial_{m}\left(\mathbb{Z}^{d-1} \times \mathbb{N}^{*}\right)$. Recall that $K_{+}(z, \gamma)$ is the Martin kernel of the Markov process $\left(Z_{+}(t)\right)$. The Martin compactification of $\mathbb{Z}^{d-1} \times \mathbb{N}^{*}$ for the Markov process $\left(Z_{+}(t)\right)$ is the unique smallest compactification of the set $\mathbb{Z}^{d-1} \times \mathbb{N}^{*}$ for which the functions

$$
z^{\prime} \rightarrow K_{+}\left(z, z^{\prime}\right)=G_{+}\left(z, z^{\prime}\right) / G_{+}\left(z_{0}, z^{\prime}\right)
$$

with a given $z_{0} \in \mathbb{Z}^{d-1} \times \mathbb{N}^{*}$ extend continuously for all $z \in \mathbb{Z}^{d-1} \times \mathbb{N}^{*}$. The mapping $\gamma \rightarrow K_{+}(z, \gamma)$ is therefore continuous on $\partial_{m}\left(\mathbb{Z}^{d-1} \times \mathbb{N}^{*}\right)$ for every $z \in \mathbb{Z}^{d-1} \times \mathbb{N}^{*}$. Moreover, for every $\gamma \in \partial_{m}\left(\mathbb{Z}^{d-1} \times \mathbb{N}^{*}\right)$, according to the definition of the minimal Martin boundary (see Woess 24]), the function $z \rightarrow K(z, \gamma)$ is a minimal harmonic function for the Markov process $\left(Z_{+}(t)\right)$ with $K\left(z_{0}, \gamma\right)=1$. By Lemma [5.5, from this it follows that

$$
K_{+}(z, \gamma)=c_{\gamma} h_{a(\gamma),+}(z), \quad \forall z \in \mathbb{Z}^{d-1} \times \mathbb{N}^{*}
$$

with some $a(\gamma) \in \partial_{+} D$ and $c_{\gamma}=1 / h_{a(\gamma),+}\left(z_{0}\right)$. Hence, for $z_{0}=\left(x_{0}, y_{0}\right)$ and $z=\left(x, y_{0}\right)$,

$$
K_{+}(z, \gamma)=\exp \left(\alpha(\gamma) \cdot\left(x-x_{0}\right)\right)
$$

where $\alpha(\gamma) \in \mathbb{R}^{d-1}$ denotes the vector of $d-1$ first coordinates of $a(\gamma)$. Since the mapping $\gamma \rightarrow K_{+}(z, \gamma)$ is continuous on $\partial_{m}\left(\mathbb{Z}^{d-1} \times \mathbb{N}^{*}\right)$ for every $\mathbb{Z}^{d-1} \times \mathbb{N}^{*}$, then from the last relation it follows that the mapping $\gamma \rightarrow \alpha(\gamma) \cdot x$ is continuous on $\partial_{m}\left(\mathbb{Z}^{d-1} \times \mathbb{N}^{*}\right)$ for every $x \in \mathbb{Z}^{d-1}$ and consequently, the mapping $\gamma \rightarrow \alpha(\gamma)$ is also continuous on $\partial_{m}\left(\mathbb{Z}^{d-1} \times \mathbb{N}^{*}\right)$. From this it follows that the mapping $\gamma \rightarrow a(\gamma)$ from $\partial_{m}\left(\mathbb{Z}^{d-1} \times \mathbb{N}^{*}\right)$ to $\partial_{+} D$ is also continuous because for every $\alpha \in \mathbb{R}^{d-1}$ for which $\inf _{\beta \in \mathbb{R}} \varphi(\alpha, \beta) \leq 1$ there is a unique $\beta_{\alpha} \in \mathbb{R}$ such that $\left(\alpha, \beta_{a}\right) \in \partial_{+} D$, and the mapping $\alpha \rightarrow\left(\alpha, \beta_{\alpha}\right)$ from $\left\{\alpha \in \mathbb{R}^{d-1}: \inf _{\beta} \varphi(\alpha, \beta) \leq 1\right\}$ to $\partial_{+} D$ is continuous. The equalities (5.8) and (5.9) imply therefore (5.1) with the positive Borel measure $\nu_{h}$ on $\partial_{+} D$ defined by

$$
\nu_{h}(B)=\int_{\{\gamma: a(\gamma) \in B\}} c_{\gamma} d \tilde{\nu}_{h}(\gamma)
$$

for every Borel subset $B \subset \partial_{+} D$ (the set $\{\gamma: a(\gamma) \in B\}$ is here measurable because the mapping $\gamma \rightarrow a(\gamma)$ is continuous and hence, the measure $\nu$ is well defined). The first assertion of Proposition 5.1 is therefore proved.

Now, to prove the second assertion it is sufficient to show that a non-zero harmonic function $h \geq 0$ satisfies (5.2) for some $a=(\alpha, \beta) \in \partial_{+} D$ if and only if

$$
\operatorname{supp}\left(\nu_{h}\right)=\{a\} .
$$

By Lemma 5.4, for every $a \in \partial_{+} D$, the function $h_{a,+}$ is harmonic for the Markov process $\left(Z_{+}(t)\right)$ and satisfies (5.2) because for every $z=(x, y) \in \mathbb{Z}^{d-1} \times \mathbb{N}^{*}$,

$$
\exp (-\alpha \cdot x) h_{a,+}(x, y)=h_{a,+}(0, y)
$$


Conversely, if $\operatorname{supp}\left(\nu_{h}\right) \neq\{\hat{a}\}$ for some $\hat{a}=(\hat{\alpha}, \hat{\beta}) \in \partial_{+} D$, then there are $a_{0}=$ $\left(\alpha_{0}, \beta_{0}\right) \in \partial_{+} D$ and $\varepsilon>0$ such that $\left|\hat{a}-a_{0}\right| \geq\left|\hat{\alpha}-\alpha_{0}\right|>\varepsilon$ and

$$
\nu\left(B\left(a_{0}, \varepsilon\right) \cap \partial_{+} D\right)>0
$$

where $B\left(a_{0}, \varepsilon\right)$ denotes the open ball in $\mathbb{R}^{d}$ centered at $a_{0}$ and having the radius $\varepsilon>0$. Moreover, since for every $a=(\alpha, \beta) \in B\left(a_{0}, \varepsilon\right)$,

$$
\left|\alpha-\alpha_{0}\right| \leq\left|a-a_{0}\right|<\varepsilon<\left|\hat{\alpha}-\alpha_{0}\right|
$$

then there exists $x_{0} \in \mathbb{Z}^{d-1} \operatorname{such}$ that $(\alpha-\hat{\alpha}) \cdot x_{0}>0$ for all $a=(\alpha, \beta) \in B\left(a_{0}, \varepsilon\right)$ and consequently, using Fatou lemma we get

$$
\begin{aligned}
\sup _{x \in \mathbb{R}^{d-1}} e^{-\hat{\alpha} \cdot x} h(x, y) & \geq \limsup _{n \rightarrow \infty} e^{-n \hat{\alpha} \cdot x_{0}} h\left(n x_{0}, y\right) \\
& \geq \limsup _{n \rightarrow \infty} \int_{B\left(a_{0}, \varepsilon\right) \cap \partial_{+} D} e^{n(\alpha-\hat{\alpha}) \cdot x_{0}} h_{a,+}(0, y) d \nu_{h}(a) \\
& \geq \int_{B\left(a_{0}, \varepsilon\right) \cap \partial_{+} D} \lim _{n \rightarrow \infty} e^{n(\alpha-\hat{\alpha}) \cdot x_{0}} h_{a,+}(0, y) d \nu_{h}(a)=+\infty
\end{aligned}
$$

Hence, if a non-zero harmonic function $h \geq 0$ satisfies (5.2) for some $a=(\alpha, \beta) \in$ $\partial_{+} D$ then $\operatorname{supp}(\nu)=\{a\}$ and consequently $h=c h_{a,+}$ with some $c>0$. The second assertion of Proposition 5.1 is proved.

Finally, by Lemma [5.4 for every $a \in \partial_{+} D$, the function $h_{a,+}>0$ is harmonic for the Markov process $\left(Z_{+}(t)\right)$. Moreover, if a non-negative harmonic function $h$ satisfies the inequality $h \leq h_{a,+}$ for some $a \in \partial_{+} D$ then for $h$ the inequality (5.2) holds with the same $a \in \partial_{+} D$ and consequently $h=c h_{a,+}$ for some $c \geq 0$. For every $a \in \partial_{+} D$, the harmonic function $h_{a,+}>0$ is therefore minimal and conversely, by Lemma 5.5. every minimal harmonic function of the Markov process $\left(Z_{+}(t)\right)$ is of the form $c h_{a,+}$ with some $c>0$ and $a \in \partial_{+} D$. Proposition 5.1 is proved.

\section{Proof of Theorem 1}

The first assertion of Theorem 1 is proved by Proposition 5.1 To prove the second assertion of Theorem 1 we use Proposition 3.3 combined with Proposition 4.2 and Proposition 5.1

Consider a sequence of points $z_{n} \in \mathbb{Z}^{d-1} \times \mathbb{N}^{*}$ with $\lim _{n}\left|z_{n}\right|=\infty$ such that $\lim _{n} z_{n} /\left|z_{n}\right|=q \in \mathcal{S}_{+}^{d}$. Suppose first that $m /|m| \in \mathcal{S}_{+}^{d}$ and let $q=m /|m|$. Then $q=q(0), a(q)=0$ and by Proposition 4.2 ,

$$
\liminf _{n \rightarrow \infty} \frac{1}{\left|z_{n}\right|} \log G_{+}\left(z, z_{n}\right) \geq-a(q) \cdot q=0 .
$$

Furthermore, by Lemma 4.1] the Markov process $\left(Z_{+}(t)\right)$ satisfies the communication condition (A1'). The function

$$
\varphi(a)=\sup _{z \in \mathbb{Z}^{d-1} \times \mathbb{N}^{*}} \mathbb{E}_{z}\left(\exp \left(a \cdot\left(Z_{+}(1)-z\right)\right)\right)=\sum_{z^{\prime} \in \mathbb{Z}^{d}} \mu\left(z^{\prime}\right) \exp \left(a \cdot z^{\prime}\right)
$$

is finite everywhere on $\mathbb{R}^{d}$ and hence, the condition (A2') is also satisfied. Moreover, let $\hat{k}$ be the greatest common divisor 1 of the set

$$
\mathcal{K}=\left\{n \in \mathbb{N}^{*}: \inf _{z \in \mathbb{Z}^{d-1} \times \mathbb{N}^{*}} \mathbb{P}_{z}\left(Z_{+}(n)=z\right)>0\right\} .
$$

\footnotetext{
${ }^{1}$ Using the same arguments as in the proof of Lemma 4.1 one can easily show that $\hat{k}$ is the period of the random walk $(Z(t))$
} 
If a non-negative function $\tilde{h}: \mathbb{Z}^{d-1} \times \mathbb{N}^{*} \rightarrow \mathbb{R}_{+}$is harmonic for the Markov process $\left(Z_{+}(t)\right)$ and satisfies the equality $\tilde{h}(z+\hat{k} w)=\tilde{h}(z)$ for all $z \in \mathbb{Z}^{d-1} \times \mathbb{N}^{*}$ and $w \in \mathbb{Z}^{d-1} \times\{0\}$ then also

$$
\sup _{x \in \mathbb{Z}^{d-1}} h(x, y)=\max _{x \in \mathbb{Z}^{d-1}:|x| \leq \hat{k} d} h(x, y)<\infty
$$

and consequently, by Proposition [5.1] $\tilde{h}=c h_{0,+}$ for some $c>0$. Hence, the condition (A3) is also satisfied with $h=h_{0,+}$. The hypotheses of Proposition 3.3 are therefore satisfied and (1.3) holds.

To extend this result for an arbitrary $q \in \mathcal{S}_{+}^{d}$, it is sufficient to apply the above arguments for a twisted random walk $\tilde{Z}(t)=(\tilde{X}(t), \tilde{Y}(t))$ on $\mathbb{Z}^{d}$ with transition probabilities

$$
\tilde{p}\left(z, z^{\prime}\right)=\tilde{\mu}\left(z^{\prime}-z\right) \doteq \mu\left(z^{\prime}-z\right) \exp \left(a(q) \cdot\left(z^{\prime}-z\right)\right)
$$

for which the mean is equal to

$$
\tilde{m}=\sum_{z \in \mathbb{Z}^{d}} z \tilde{\mu}(z)=\nabla \varphi(a(q)) .
$$

For such a random walk, $q=\tilde{m} /|\tilde{m}| \in \mathcal{S}_{+}^{d}$ and the hypotheses (A) are satisfied if they are satisfied for the initial random walk $(Z(t))$. Hence, using the same arguments as above we conclude that Green's function $\tilde{G}_{+}\left(z, z^{\prime}\right)$ of the random walk $\left(\tilde{Z}_{+}(t)\right)$ killed outside of the half-space $\mathbb{Z}^{d-1} \times \mathbb{N}^{*}$ satisfies the following property : for every $z \in \mathbb{Z}^{d-1} \times \mathbb{N}^{*}$,

$$
\lim _{n \rightarrow \infty} \tilde{G}_{+}\left(z, z_{n}\right) / \tilde{G}_{+}\left(z_{0}, z_{n}\right)=\tilde{h}_{0,+}(z) / \tilde{h}_{0,+}\left(z_{0}\right)
$$

where

$$
\tilde{h}_{0,+}(z) \doteq \begin{cases}y-\mathbb{E}_{z}(\tilde{Y}(\tau), \tau<+\infty) & \text { if } \tilde{m} \in \mathbb{R}^{d-1} \times\{0\}, \\ \mathbb{P}_{z}(\tau=+\infty) & \text { if } \left.\tilde{m} \in \mathbb{R}^{d-1} \times\right] 0,+\infty[\end{cases}
$$

with $\tau=\inf \{t \geq 0: \tilde{Y}(t) \leq 0\}$. Finally, using the identities

$$
\tilde{G}_{+}\left(z, z^{\prime}\right)=e^{a(q) \cdot\left(z^{\prime}-z\right)} G_{+}\left(z, z^{\prime}\right) \quad \text { and } \quad \tilde{h}_{0,+}(z)=e^{-a(q) \cdot z} h_{a(q),+}(z)
$$

we get (1.3). Theorem 1 is therefore proved.

\section{Application for homogeneous RANDOM WALK}

In the present section, the Martin boundary of a homogeneous random walk on $\mathbb{Z}^{d}$ is obtained as a consequence of Proposition 3.3 .

Remark that a random walk $Z(t)$ on $\mathbb{Z}^{d}$ with transition probabilities $p\left(z, z^{\prime}\right)=$ $\mu\left(z^{\prime}-z\right), z, z^{\prime} \in \mathbb{Z}^{d}$, can be considered as a Markov additive process where the additive part is $A(t)=Z(t)$ and the Markovian part is constant $M(t) \equiv 0$ with $E=\{0\}$. In this setting, Proposition 3.3 implies the following result.

Theorem [Ney and Spitzer [18] Suppose that the random walk $Z(t)$ is irreducible with a non zero mean

$$
m=\sum_{z \in \mathbb{Z}^{d}} z \mu(z) \neq 0,
$$

and let the jump generating function $\varphi$ of (1.1) be finite in a neighborhood of the set $D=\left\{a \in \mathbb{R}^{d}: \varphi(a) \leq 1\right\}$. Then for any $z \in \mathbb{Z}^{d}$, uniformly on $q \in \mathcal{S}^{d}$,

$$
G\left(z, z_{n}\right) / G\left(0, z_{n}\right) \rightarrow \exp (a(q) \cdot z)
$$


when $\left|z_{n}\right| \rightarrow \infty$ and $z_{n} /\left|z_{n}\right| \rightarrow q$

Proof. Indeed, under the hypotheses of this theorem, the Markov additive process $(A(t), M(t))$ with the additive part $A(t)=Z(t)$ and the constant Markovian part $M(t) \equiv 0$ satisfies the conditions (A1') and (A2') (see the proof of Lemma 4.1 for more details). Hence, by Proposition 3.3 , for any sequence of points $z_{n} \in \mathbb{Z}^{d}$ with $\lim _{n}\left|z_{n}\right|=\infty$, if

$$
\liminf _{n \rightarrow \infty} \frac{1}{\left|z_{n}\right|} \log G\left(0, z_{n}\right) \geq 0
$$

then also

$$
\lim _{n \rightarrow \infty} G\left(z+\hat{k} w, z_{n}\right) / G\left(z, z_{n}\right)=1, \quad \forall z, w \in \mathbb{Z}^{d}
$$

where $\hat{k}$ is the period of the random walk $(Z(t))$. Moreover, the same arguments as in the proof of Proposition 4.21 show that

$$
\liminf _{n \rightarrow \infty} \frac{1}{\left|z_{n}\right|} \log G\left(0, z_{n}\right) \geq-a(q) \cdot q
$$

when $\left|z_{n}\right| \rightarrow \infty$ and $z_{n} /\left|z_{n}\right| \rightarrow q \in \mathcal{S}^{d}$. Consider now a sequence of points $z_{n} \in \mathbb{Z}^{d}$ such that $\left|z_{n}\right| \rightarrow \infty$ and $z_{n} /\left|z_{n}\right| \rightarrow q=q(0) \in \mathcal{S}^{d}$ as $n \rightarrow \infty$. Then $a(q)=0$ and consequently, the last inequality provides the inequality (7.1). Hence, the equality (7.2) holds. Moreover, let for a subsequence $\left(z_{n_{k}}\right)$, the sequence of functions $G\left(\cdot, z_{n_{k}}\right) / G\left(0, z_{n_{k}}\right)$ converge pointwise in $\mathbb{Z}^{d}$ and let

$$
h(z)=\lim _{k \rightarrow \infty} G\left(z, z_{n_{k}}\right) / G\left(0, z_{n_{k}}\right) .
$$

Then by Fatou lemma, the function $h$ is super-harmonic for $(Z(t))$ :

$$
\mathbb{E}_{z}(h(Z(t))) \leq h(z), \quad \forall t \in \mathbb{N}, z \in \mathbb{Z}^{d}
$$

and from (7.2) it follows that $h(z+\hat{k} w)=h(z)$ for all $z, w \in \mathbb{Z}^{d}$. Hence, $h$ is a super-harmonic function with a finite number of values. By Minimum principal (see Woess 24]) from this it follows that the function $h$ is constant. Moreover, since $h(0)=1$ we conclude that $h(z)=1$ for all $z \in \mathbb{Z}^{d}$ and consequently,

$$
G\left(z, z_{n_{k}}\right) / G\left(0, z_{n_{k}}\right) \rightarrow 1, \quad \forall z \in \mathbb{Z}^{d}
$$

when $\left|z_{n}\right| \rightarrow \infty$ and $z_{n} /\left|z_{n}\right| \rightarrow q(0)$ for any subsequence $\left(z_{n_{k}}\right)$ for which the sequence of functions $G\left(\cdot, z_{n_{k}}\right) / G\left(0, z_{n_{k}}\right)$ converges pointwise in $\mathbb{Z}^{d}$. This proves that

$$
G\left(z, z_{n}\right) / G\left(0, z_{n}\right) \rightarrow 1, \quad \forall z \in \mathbb{Z}^{d}
$$

when $\left|z_{n}\right| \rightarrow \infty$ and $z_{n} /\left|z_{n}\right| \rightarrow q(0)$ because for every $z \in \mathbb{Z}^{d}$, the sequence $G\left(z, z_{n}\right) / G\left(0, z_{n}\right)$ is bounded .

Suppose now that $\left|z_{n}\right| \rightarrow \infty$ and $z_{n} /\left|z_{n}\right| \rightarrow q \neq q(0)$ as $n \rightarrow \infty$. Then the same arguments applied for the twisted random walk having transition probabilities $\tilde{p}\left(z, z^{\prime}\right)=\exp \left(a(q) \cdot\left(z^{\prime}-z\right)\right) \mu\left(z^{\prime}-z\right)$, the mean $m(q)=\nabla \varphi(a(q))$ and Green's function $\tilde{G}\left(z, z^{\prime}\right)=\exp \left(a(q) \cdot\left(z^{\prime}-z\right)\right) G\left(z, z^{\prime}\right)$ show that $\tilde{G}\left(z, z_{n}\right) / \tilde{G}\left(0, z_{n}\right) \rightarrow 1$ as $n \rightarrow \infty$ for every $z \in \mathbb{Z}^{d}$ and consequently

$$
\lim _{n \rightarrow \infty} G\left(z, z_{n}\right) / G\left(0, z_{n}\right)=\exp (a(q) \cdot z), \quad \forall z \in \mathbb{Z}^{d} .
$$

\footnotetext{
${ }^{2}$ Here, instead of Proposition 4.1 one should use Mogulskii's theorem
} 
Consider now the mapping $z \rightarrow w(z)=z /(1+|z|)$ from $\mathbb{Z}^{d}$ to $\mathcal{R}=\{w=z /(1+|z|)$ : $\left.z \in \mathbb{Z}^{d}\right\}$ and its inverse mapping $w \rightarrow z(w)=w /(1-|w|)$. Remark that the boundary $\partial \mathcal{R}$ of the set $\mathcal{R}$ in $\mathbb{R}^{d}$ is the $d$-dimensional sphere $\mathcal{S}^{d}$, the closure $\overline{\mathcal{R}}$ of the set $\mathcal{R}$ is compact and for every $z \in \mathbb{Z}^{d}$, the function

$$
f_{z}(w)= \begin{cases}G(z, z(w))) / G(0, z(w))) & \text { if } w \in \mathcal{R}, \\ \exp (a(w) \cdot z) & \text { if } w \in \partial \mathcal{R}\end{cases}
$$

is continuous on $\overline{\mathcal{R}}$. Hence, for every $z \in \mathbb{Z}^{d}$, the function $f_{z}$ is uniformly continuous on $\overline{\mathcal{R}}$ and consequently, for any $\varepsilon>0$ there is $\delta>0$ such that for all $q \in \mathcal{S}^{d}$ and $w \in \mathcal{R}$,

$$
\left.\left.\mid G\left(z, z_{n}\right)\right) / G\left(0, z_{n}\right)\right)-\exp (a(q) \cdot z) \mid<\varepsilon
$$

whenever $\left|q-w\left(z_{n}\right)\right|<\delta$. This proves that $G\left(z, z_{n}\right) / G\left(0, z_{n}\right) \rightarrow \exp (a(q) \cdot z)$ when $\left|z_{n}\right| \rightarrow \infty$ and $z_{n} /\left|z_{n}\right| \rightarrow q$ uniformly on $q \in \mathcal{S}^{d}$ for every $z \in \mathbb{Z}^{d}$.

\section{Proof of Proposition 3.2}

To prove this proposition it is sufficient to show that

$$
\limsup _{n \rightarrow \infty} \mathcal{G}\left(z+w^{\prime}, z_{n}\right) / \mathcal{G}\left(z+w, z_{n}\right) \leq 1
$$

for any $z \in \mathbb{Z}^{d} \times E$. The main idea of the proof is the following : Remark first of all that if (3.4) holds for some $z_{0} \in \mathbb{Z}^{d} \times E$ then also

$$
\liminf _{n \rightarrow \infty} \frac{1}{\left|z_{n}\right|} \log \mathcal{G}\left(z, z_{n}\right) \geq 0
$$

for all $z \in \mathbb{Z}^{d} \times E$ because the Markov-process $\mathcal{Z}(t)$ is irreducible. This inequality shows that the terms of the order $c \exp \left(-\delta\left|z_{n}\right|\right)$ give an asymptotically negligible contribution to $\mathcal{G}\left(z, z_{n}\right)$. The quantities $\mathcal{G}\left(z, z_{n}\right)$ are decomposed into a negligible part of the order $c \exp \left(-\delta\left|z_{n}\right|\right)$ and a main part for which it is possible to apply the method of Bernoulli part decomposition developed in [5, 7, 17.

The first step of the proof shows that there is $\kappa>0$ for which the part

$$
\sum_{0 \leq t \leq \kappa\left|z_{n}\right|} \mathbb{P}_{z}\left(\mathcal{Z}(t)=z_{n}\right)
$$

of $\mathcal{G}\left(z, z_{n}\right)$ is negligible. Under the hypotheses of our lemma, there is $\delta_{0}>0$ such that for any $0<\delta \leq \delta_{0}$,

$$
C_{\delta} \doteq \sup _{a \in \mathbb{R}^{d+k}:|a| \leq \delta} \sup _{z \in \mathbb{Z}^{d} \times E} \mathbb{E}_{z}\left(e^{a \cdot(\mathcal{Z}(1)-z)}\right)<\infty
$$

and hence, using Chebychev's inequality and Markov property we obtain that for any $l>0, z \in \mathbb{Z}^{d} \times E$ and any $a \in \mathbb{R}^{d} \times \mathbb{R}^{k}$ with $|a| \leq \delta \leq \delta_{0}$, the following relation holds

$$
\mathbb{P}_{z}(a \cdot \mathcal{Z}(t) \geq l) \leq e^{-l} \mathbb{E}_{z}\left(e^{a \cdot \mathcal{Z}(t)}\right) \leq C_{\delta}^{t} e^{-l+a \cdot z} \leq C_{\delta}^{t} e^{-l+\delta|z|}, \quad \forall t \in \mathbb{N} .
$$

Using this inequality with $l=\delta\left|z_{n}\right|$ for $0<\delta \leq \delta_{0}$ we obtain

$$
\mathbb{P}_{z}\left(\mathcal{Z}(t)=z_{n}\right) \leq P_{z}\left(z_{n} \cdot \mathcal{Z}(t)=\left|z_{n}\right|^{2}\right) \leq C_{\delta}^{t} \exp \left(-\delta\left|z_{n}\right|+\delta|z|\right)
$$


and hence, for $\kappa=\delta /\left(2 \ln C_{\delta}\right)$ we get

$$
\begin{aligned}
\sum_{0 \leq t \leq \kappa\left|z_{n}\right|} \mathbb{P}_{z}\left(\mathcal{Z}(t)=z_{n}\right) & \leq \exp \left(-\delta\left|z_{n}\right|+\delta|z|\right) \sum_{0 \leq t \leq \kappa\left|z_{n}\right|} C_{\delta}^{t} \\
& \leq \exp \left(-\frac{\delta}{2}\left|z_{n}\right|+\delta|z|\right) /\left(C_{\delta}-1\right)
\end{aligned}
$$

for any $z \in \mathbb{Z}^{d} \times E$ and any $n \in \mathbb{N}$. The left hand side of this inequality is therefore a negligible part of $\mathcal{G}\left(z, z_{n}\right)$.

Next, the method of Bernoulli part decomposition is applied. Recall that under the hypotheses of our proposition, for given $w, w^{\prime} \in \mathbb{Z}^{d} \times\{0\}$, there are $\varepsilon>0$ and $\hat{n} \in \mathbb{N}^{*}$ such that

$$
\inf _{z \in \mathbb{Z}^{d} \times E} \min \left\{p^{(\hat{n})}(z, z+w), p^{(\hat{n})}\left(z, z+w^{\prime}\right)\right\} \geq \varepsilon .
$$

Suppose first that $\hat{n}=1$ and let us consider two independent sequences $(\xi(t))$ and $(\zeta(t))$ of independent Bernoulli random variables with means $\mathbb{E}(\xi(t))=\varepsilon$ and $\mathbb{E}(\zeta(t))=1 / 2$. Then the Markov additive process $\mathcal{Z}(t)=(A(t), M(t))$ can be represented in the following way : at time $t$ we pick a random variable $\xi(t)$ and if $\xi(t)=1$, then we let $\mathcal{Z}(t+1)=\mathcal{Z}(t)+\zeta(t) w+(1-\zeta(t)) w^{\prime}$. Otherwise, for each $y \in E$, we put $M(t+1)=y$ with probability

$$
\left(p_{M}(M(t), y) 1_{\{M(t) \neq y\}}+\left(p_{M}(y, y)-\varepsilon\right) 1_{\{M(t)=y\}}\right) /(1-\varepsilon)
$$

where

$$
p_{M}\left(y^{\prime}, y\right) \doteq \sum_{x \in \mathbb{Z}^{d}} p\left(\left(0, y^{\prime}\right),(x, y)\right)
$$

and we let

$$
\begin{aligned}
A(t+1)=A(t)+\sum_{y \in E} 1_{\{M(t)=M(t+1)=y\}} B_{y, y}(t) & \\
& +\sum_{y, y^{\prime} \in E: y \neq y^{\prime}} 1_{\left\{M(t)=y, M(t+1)=y^{\prime}\right\}} B_{y, y^{\prime}}(t)
\end{aligned}
$$

where $\left(B_{y, y^{\prime}}(t), t \in \mathbb{N}, y \in E\right)$ is a family of mutually independent random variables which are independent of the Markov process $(M(t))$ and of the sequences $(\xi(t))$ and $(\zeta(t))$, such that for $x \in \mathbb{Z}^{d}$ and $y, y^{\prime} \in E$,

$$
\begin{gathered}
\mathbb{P}\left(B_{y, y^{\prime}}(t)=x\right)=p\left((0, y),\left(x, y^{\prime}\right)\right) / p_{M}\left(y, y^{\prime}\right) \quad \text { when } \quad y \neq y^{\prime}, \\
\mathbb{P}\left(B_{y, y}(t)=x\right)=\frac{p((0, y),(x, y))}{(1-\varepsilon) p_{M}(y, y)} \quad \text { if } \quad(x, 0) \notin\left\{w, w^{\prime}\right\},
\end{gathered}
$$

and

$$
\mathbb{P}\left(B_{y, y}(t)=x\right)=\frac{p((0, y),(x, y))-\varepsilon / 2}{(1-\varepsilon) p_{M}(y, y)} \text { for } \quad(x, 0) \in\left\{w, w^{\prime}\right\} .
$$

This representation shows that

$$
\mathcal{Z}(t)=(A(t), M(t))=\left(Q(t)+\sum_{s=1}^{N_{t}}\left(\zeta(s) w+(1-\zeta(s)) w^{\prime}\right), M(t)\right)
$$

where $N_{t}=\operatorname{Card}\{s \in \mathbb{N}: s \leq t, \xi(s)=1\}$ is a Binomial random variable with mean $\varepsilon t$ and variance $\varepsilon(1-\varepsilon) t$, the random vector $\left(Q(t), M(t), N_{t}\right)$ is independent 
of the sequence $(\zeta(s))$ (the random variables $Q(t), M(t)$ and $N_{t}$ are dependent) and the equality holds in a sense of the identity of the distributions. Hence, letting

$$
L_{n}=\sum_{s=1}^{n} \zeta(s)
$$

we obtain

$$
\begin{aligned}
\mathbb{P}_{z} & \left(\mathcal{Z}(t)=z_{n}, N_{t}=N, L_{N}=l\right) \\
\quad & =\mathbb{P}_{z}\left((Q(t), M(t))=z_{n}-l w-(N-l) w^{\prime}, N_{t}=N, L_{N}=l\right) \\
\quad & \mathbb{P}_{z}\left((Q(t), M(t))=z_{n}-l w-(N-l) w^{\prime}, N_{t}=N\right) \mathbb{P}\left(L_{N}=l\right)
\end{aligned}
$$

for all $z \in \mathbb{Z}^{d} \times E, n \geq 0$ and $0 \leq l \leq N$. Furthermore, by Chebychev's inequality,

$$
\mathbb{P}\left(N_{t}<\varepsilon t / 2\right) \leq \inf _{\eta<0} e^{-\eta \varepsilon t / 2} \mathbb{E}\left(e^{\eta N_{t}}\right)=\exp \left(-t \theta_{1}\right)
$$

where $\theta_{1} \doteq \sup _{\eta<0}\left(\eta \varepsilon / 2-\log \left(\varepsilon e^{\eta}+1-\varepsilon\right)\right)>0$ because the function $f_{1}(\eta)=$ $\eta \varepsilon / 2-\log \left(\varepsilon e^{\eta}+1-\varepsilon\right)$ is concave, $f_{1}(0)=0$ and $f_{1}^{\prime}(0)=-\varepsilon / 2<0$. From this it follows that

$$
\begin{aligned}
\sum_{t>\kappa\left|z_{n}\right|} \mathbb{P}_{z}\left(\mathcal{Z}(t)=z_{n}, N_{t}<\varepsilon t / 2\right) & \leq \sum_{t>\kappa\left|z_{n}\right|} \mathbb{P}\left(N_{t}<\varepsilon t / 2\right) \\
& \leq \exp \left(-\kappa \theta_{1}\left|z_{n}\right|\right) /\left(1-\exp \left(-\theta_{1}\right)\right)
\end{aligned}
$$

Hence, the left hand side of this inequality is a negligible part of $\mathcal{G}\left(z, z_{n}\right)$. Moreover, for $0<\sigma<1 / 2$,

$$
\begin{aligned}
\mathbb{P}\left(\left|L_{N}-\frac{N}{2}\right|>\sigma N\right) & =2 \mathbb{P}\left(L_{N}>\frac{N}{2}+\sigma N\right) \\
& \leq 2 \inf _{\eta>0} e^{-\eta(1 / 2+\sigma) N} \mathbb{E}\left(\exp \left(\eta \sum_{s=1}^{N} \zeta(s)\right)\right) \\
& \leq 2 \exp \left(-N \theta_{2}\right)
\end{aligned}
$$

where $\theta_{2} \doteq \sup _{\eta>0}\left(\eta(1 / 2+\sigma)-\log \left(\left(e^{\eta}+1\right) / 2\right)\right)>0$ because the function $f_{2}(\eta)=\eta(1 / 2+\sigma)-\log \left(\left(e^{\eta}+1\right) / 2\right)$ is concave, $f_{2}(0)=0$ and $f_{2}^{\prime}(0)=\sigma>0$. Using this inequality we get

$$
\begin{aligned}
\sum_{t>\kappa\left|z_{n}\right|} & \mathbb{P}_{z}\left(\mathcal{Z}(t)=z_{n}, N_{t} \geq \varepsilon t / 2,\left|L_{N_{t}}-N_{t} / 2\right|>\sigma N_{t}\right) \\
& \leq \sum_{t>\kappa\left|z_{n}\right|} \sum_{N \geq \varepsilon t / 2} \mathbb{P}\left(N_{t}=N,\left|L_{N}-N / 2\right|>\sigma N\right) \\
& \leq 2 \sum_{t>\kappa\left|z_{n}\right|} \sum_{N \geq \varepsilon t / 2} \exp \left(-N \theta_{2}\right) \leq \sum_{t>\kappa\left|z_{n}\right|} \exp \left(-\varepsilon \theta_{2} t / 2\right) /\left(1-\exp \left(-\theta_{2}\right)\right) \\
& \leq \exp \left(-\varepsilon \theta_{2} \kappa\left|z_{n}\right| / 2\right) /\left(1-\exp \left(-\theta_{2}\right)\right)\left(1-\exp \left(-\varepsilon \theta_{2} / 2\right)\right) .
\end{aligned}
$$

The last inequality shows that the part

$$
\sum_{t>\kappa\left|z_{n}\right|} \mathbb{P}_{z}\left(\mathcal{Z}(t)=z_{n}, N_{t} \geq \varepsilon t / 2,\left|L_{N_{t}}-N_{t} / 2\right|>\sigma N_{t}\right)
$$


of $\mathcal{G}\left(z, z_{n}\right)$ is also negligible. Finally, remark that for all those $l \in \mathbb{N}$ for which $|l-N / 2| \leq \sigma N$, the following relation holds

$$
\mathbb{P}\left(L_{N}=l\right)=\mathbb{P}\left(L_{N}=l+1\right) \frac{l+1}{N-l} \leq \mathbb{P}\left(L_{N}=l+1\right) \frac{1+2 \sigma+2 / N}{1-2 \sigma}
$$

and hence, using (8.5) we obtain

$$
\begin{aligned}
& \mathbb{P}_{z}\left(\mathcal{Z}(t)=z_{n}, N_{t}=N, L_{N}=l\right) \\
& =\mathbb{P}_{z}\left((Q(t), M(t))=z_{n}-l w-(N-l) w^{\prime}, N_{t}=N\right) \mathbb{P}\left(L_{N}=l\right) \\
& \leq \frac{1+2 \sigma+2 / N}{1-2 \sigma} \mathbb{P}_{z}\left((Q(t), M(t))=z_{n}-l w-(N-l) w^{\prime}, N_{t}=N, L_{N}=l+1\right) \\
& \leq \frac{1+2 \sigma+2 / N}{1-2 \sigma} \mathbb{P}_{z}\left(\mathcal{Z}(t)=z_{n}+w-w^{\prime}, N_{t}=N, L_{N}=l+1\right)
\end{aligned}
$$

From the last inequality it follows that

$$
\begin{aligned}
\sum_{t>\kappa\left|z_{n}\right|} \mathbb{P}_{z}(\mathcal{Z}(t) & \left.=z_{n}, N_{t} \geq \varepsilon t / 2,\left|L_{N_{t}}-N_{t} / 2\right| \leq \sigma N_{t}\right) \\
& \leq \frac{1+2 \sigma+4 /\left(\varepsilon \kappa\left|z_{n}\right|\right)}{1-2 \sigma} \sum_{t>\kappa\left|z_{n}\right|} \mathbb{P}_{z}\left(\mathcal{Z}(t)=z_{n}-w+w^{\prime}\right) \\
& \leq \frac{1+2 \sigma+4 /\left(\varepsilon \kappa\left|z_{n}\right|\right)}{1-2 \sigma} \mathcal{G}\left(z, z_{n}-w+w^{\prime}\right)
\end{aligned}
$$

where $\mathcal{G}\left(z, z_{n}-w+w^{\prime}\right)=\mathcal{G}\left(z+w-w^{\prime}, z_{n}\right)$ because $w, w^{\prime} \in \mathbb{Z}^{d} \times\{0\}$. Using the above inequality together with (8.3), (8.6) and (8.7) we conclude that for any $0<\delta<\delta_{0}$ and $0<\sigma<1 / 2$ there are $\kappa>0, C>0$ and $\theta>0$ such that

$$
\mathcal{G}\left(z, z_{n}\right) \leq \frac{1+2 \sigma+C /\left|z_{n}\right|}{1-2 \sigma} \mathcal{G}\left(z+w-w^{\prime}, z_{n}\right)+C \exp \left(-\theta\left|z_{n}\right|+\delta|z|\right) .
$$

for all $n \in \mathbb{N}$ and $z \in \mathbb{Z}^{d} \times E$. Because of (8.2) from this it follows that

$$
\limsup _{n \rightarrow \infty} \mathcal{G}\left(z, z_{n}\right) / \mathcal{G}\left(z+w-w^{\prime}, z_{n}\right) \leq \frac{1+2 \sigma}{1-2 \sigma}, \quad \forall z \in \mathbb{Z}^{d} \times E
$$

and consequently, also

$$
\limsup _{n \rightarrow \infty} \mathcal{G}\left(z+w^{\prime}, z_{n}\right) / \mathcal{G}\left(z+w, z_{n}\right) \leq \frac{1+2 \sigma}{1-2 \sigma}, \quad \forall z \in \mathbb{Z}^{d} \times E
$$

(to get the last inequality it is sufficient to replace $z$ by $z+w^{\prime}$ ). Since $0<\sigma<1 / 2$ is arbitrary, then letting at the last inequality $\sigma \rightarrow 0$ we get (8.1). When (8.4) holds with $\hat{n}=1$, the inequality (8.1) is therefore verified.

Suppose now that $\hat{n}>1$. Then for the Green's function $\tilde{\mathcal{G}}\left(z, z_{n}\right)$ of the embedded Markov chain $\tilde{\mathcal{Z}}(t)=(A(\hat{n} t), M(\hat{n} t))$ the above arguments show that for any $0<$ $\delta \leq \delta_{0}$ and $0<\sigma<1 / 2$ there are $\kappa>0, C>0, \theta>0$ such that

$$
\tilde{\mathcal{G}}\left(z, z_{n}\right) \leq \frac{1+2 \sigma+C /\left|z_{n}\right|}{1-2 \sigma} \tilde{\mathcal{G}}\left(z+w-w^{\prime}, z_{n}\right)+C \exp \left(-\theta\left|z_{n}\right|+\delta|z|\right) .
$$

for all $z \in \mathbb{Z}^{d} \times E$. Using the identities

$$
\mathcal{G}\left(z, z_{n}\right)=\sum_{t=0}^{\hat{n}-1} \sum_{z^{\prime}} p^{(t)}\left(z, z^{\prime}\right) \tilde{\mathcal{G}}\left(z^{\prime}, z_{n}\right)
$$


and

$$
\mathcal{G}\left(z+w-w^{\prime}, z_{n}\right)=\sum_{t=0}^{\hat{n}-1} \sum_{z^{\prime}} p^{(t)}\left(z, z^{\prime}\right) \tilde{\mathcal{G}}\left(z^{\prime}+w-w^{\prime}, z_{n}\right)
$$

it follows therefore that

$$
\begin{aligned}
\mathcal{G}\left(z, z_{n}\right) \leq \frac{1+2 \sigma+C /\left|z_{n}\right|}{1-2 \sigma} \mathcal{G}(z+w- & \left.w^{\prime}, z_{n}\right)+ \\
& C \sum_{t=0}^{\hat{n}-1} \sum_{z^{\prime}} p^{(t)}\left(z, z^{\prime}\right) \exp \left(\delta\left|z^{\prime}\right|-\theta\left|z_{n}\right|\right)
\end{aligned}
$$

Since under the hypotheses $\left(A_{2}\right)$, for $0<\delta \leq \delta_{0}$,

$$
\sum_{z^{\prime}} p^{(t)}\left(z, z^{\prime}\right) \exp \left(\delta\left|z^{\prime}\right|\right)<\infty
$$

then the inequality (8.8) combined with (8.2) proves (8.1). Proposition 3.2 is proved.

\section{REFERENCES}

1. L. Alili and R. A. Doney, Martin boundaries associated with a killed random walk, Ann. Inst. H. Poincaré Probab. Statist. 37 (2001), no. 3, 313-338.

2. Patrick Billingsley, Convergence of probability measures, Wiley series in probability and mathematical statistics, John Wiley \& Sons Ltd, New York, 1968.

3. A.A. Borovkov and A.A Mogulskii, The second rate function and the asymptotic problems of renewal and hitting the boundary for multidimensional random walks, Siberian Math Journal 33 (1992), no. 4, 745-782.

4. P. Cartier, Fonctions harmoniques sur un arbre, Symposia Mathematica 9 (1972), 203-270.

5. B. Davis and D. McDonald, An elementary proof of the local central limit theorem., J.Theoret.Probab. 8 (1995), 693-701.

6. Amir Dembo and Ofer Zeitouni, Large deviations techniques and applications, SpringerVerlag, New York, 1998.

7. Robert D.Foley and David R.McDonald, Bridges and networks: exact asymptotics, Ann. Appl. Probab. 15 (2005), no. 1B, 542-586.

8. R. A. Doney, The Martin boundary and ratio limit theorems for killed random walks, J.London Math.Soc. 2 (1998), no. 58, 761-768.

9. J. L. Doob, Discrete potential theory and boundaries, J.Math. and Mech. 8 (1959), 433-458.

10. E.B. Dynkin, Boundary theory of Markov processes, Russian Mathematical Surveys 24 (1969), no. $7,1-42$.

11. P.L. Hennequin, Processus de Markoff en cascade, Ann. Inst. H. Poincaré 18 (1963), no. 2, 109-196.

12. G. A. Hunt, Markoff chains and Martin boundaries, Illinois J. Math. 4 (1960), 313-340.

13. Irina Ignatiouk-Robert, Sample path large deviations and convergence parameters, Annals of Applied Probability 11 (2001), no. 4, 1292-1329.

14. - Martin boundary of a reflected random walk on a half-space, 2006, preprint: http://arxiv.org/abs/math.PR/0610242

15. I.A. Kurkova and V.A. Malyshev, Martin boundary and elliptic curves., Markov Processes Related Fields 4 (1998), 203-272.

16. R.S. Martin, Minimal positive harmonic functions, Trans. Amer. Math. Soc. 49 (1941), 137172 .

17. D. McDonald, On local limit theorems for integer valued random variables., Theory Probab. Appl. 24 (1979), 613-619.

18. P. Ney and Spitzer F, The Martin boundary for random walk, Trans. Amer. Math. Soc. (1966), no. $121,116-132$.

19. R. Tyrrell Rockafellar, Convex analysis, Princeton University Press, Princeton, NJ, 1997, Reprint of the 1970 original, Princeton Paperbacks.

20. L. C. G. Rogers and David Williams, Diffusions, Markov processes, and martingales. Vol. 1: Foundations, second ed., John Wiley \& Sons Ltd., Chichester, 1994. 
21. Walter Rudin, Real and complex analysis, McGraw-Hill series in higher mathematics, McGraw-Hill book company, Ney-York, 1974.

22. E. Seneta, Nonnegative matrices and Markov chains, second ed., Springer-Verlag, New York, 1981.

23. F. Spitzer, Principles of random walk, D. van Nostrand Company, INC, 1964.

24. Wolfgang Woess, Random walks on infinite graphs and groups, Cambridge University Press, Cambridge, 2000.

Université de Cergy-Pontoise, Département de mathématiques, 2, Avenue Adolphe Chauvin, 95302 Cergy-Pontoise Cedex, France

E-mail address: Irina.Ignatiouk@math.u-cergy.fr 